\title{
THE PRENATAL DEVELOPMENT OF THE CAT'S RETINOGENICULATE PATHWAY ${ }^{1}$
}

\author{
CARLA J. SHATZ \\ Department of Neurobiology, Stanford University School of Medicine, Stanford, California 94305
}

Received August 18, 1982; Accepted October 8, 1982

\begin{abstract}
The prenatal development of connections between the retina and the lateral geniculate nucleus (LGN) was studied by means of the anterograde axonal transport of ${ }^{3} \mathrm{H}$-amino acids or horseradish peroxidase injected intraocularly in fetal cats older than embryonic day 27 (E27) and in newborn cats. (Gestation is 65 days.) A retinothalamic pathway exists as early as E28, when label can be seen in both ipsilateral and contralateral optic tracts. Afferents from the contralateral eye are the first to invade the anlage of the LGN by E32 with those from the ipsilateral eye following about 3 days later. Initially, the pattern of labeling within the nucleus is uniform, suggesting that the two sets of afferents must share a good deal of territory at early ages. By E47, however, gaps appear in the labeling pattern contralaterally, indicating that afferents from the two eyes are beginning to segregate from cach other. Segregation continues so that by E54 it is possible to identify unambiguously regions of the LGN destined to comprise ipsilateral and contralateral eye layers. By birth, afferent input appears adult-like in organization, with the two sets of afferents almost completely segregated from each other into their appropriate layers. Cellular lamination of the nucleus has just commenced, however, thereby lagging the onset of afferent segregation by about 2 weeks. Prenatal development could be followed much more easily in the horizontal than in the coronal plane of section due to the finding here that the LGN is displaced approximately $90^{\circ}$ in the horizontal plane between E40 and E60.

Measurements of the area occupied by the ipsilateral and contralateral afferents within the LGN indicated that even prior to segregation, the two sets of afferents are not completely intermixed within the LGN. On the contrary, those from the contralateral eye retain almost exclusive control of some territory throughout development. This detail contrasts with development in primates, in which intermixing of afferents from the two eyes is thought to be complete early on (Rakic, P. (1976) Nature 261: 467-471). Nevertheless, in the cat, as in other mammals, development of the retinogeniculate pathway is broadly characterized by an initial period of overlap followed by a period of segregation that gives rise to the adult pattern of afferent input.
\end{abstract}

One attribute of the adult central nervous system is that connections within it are organized with great precision. In the visual system of higher mammals precision is manifested in the strict segregation of inputs from the two eyes at the level of both the lateral geniculate nucleus (LGN) and the primary visual cortex. The LGN consists of several cell layers interleaved by an acellular plexus, and the axons arising from the retinal ganglion cells of

\footnotetext{
'I wish to thank Angela DiBerardino and Mark Siegel for their excellent technical assistance, Drs. R. W. Guillery, P. A. Kirkwood and M. B. Luskin for their thorough and thoughtful critique of the manuscript, Dr. P. Rakic for training in the methods of fetal surgery, and Cecele Thomas for her secretarial finesse. I would also like to acknowledge gratefully the essential support provided by the National Institutes of Health (Grants EY 02858 and BRSG RR5353), the March of Dimes (Grant 5-252), and the A. P. Sloan Foundation (Grant BR2041).
}

each eye terminate in alternate layers (Minkowski, 1920 Hayhow, 1958). In the cat, afferents from the contralateral eye innervate layers $\mathrm{A}, \mathrm{C}$, and $\mathrm{C} 2$; those from the ipsilateral eye terminate in layers A1 and C1 (Guillery, 1970; Hickey and Guillery, 1973). The relay neurons of the LGN, in turn, send their axons principally to layer 4 of the visual cortex where afferents representing the right and left eyes are again segregated from each other into adjacent patches roughly $500 \mu \mathrm{m}$ wide (Hubel et al., 1977; Shatz et al., 1977).

The question arises how such a segregated state is established during the development of the visual system. A good deal is now known about the time course and pattern of development of eye dominance within layer 4 at the cortical level, both in monkeys (Rakic, 1976; LeVay et al., 1980) and in cats (LeVay et al., 1978). Perhaps the 
most important point to emerge is that in these species the adult pattern of connections only appears gradually, due to the progressive segregation of initially overlapping sets of afferents from each eye within layer 4 . Such a progression is likely to occur also at the geniculate level. For instance, in the rhesus monkey, Rakic (1979) has shown autoradiographically by means of intraocular injections of $\left[{ }^{3} \mathrm{H}\right]$ proline and fucose that by embryonic day 68 (E68), roughly 100 days before birth and the earliest age successfully studied to date, substantial connections exist between the retina and LGN. At this age, afferents from both eyes appear to occupy fully the anlage of the LGN. Then, during the next 2 months, afferents segregate from each other until the adult-like pattern of retinogeniculate projection is produced by E144.

This remarkable process by which initially overlapping inputs are segregated from one another is not unique to the visual system of mammals with extensive binocular vision. A similar process is seen in the development of the retinogeniculate pathway in ferrets (Linden et al., 1981) and rodents (So et al., 1978; Bunt et al., 1981). Presumably, it is differences in the details of the process from species to species that account for the adult pattern characteristic of each. One noteworthy difference might reside in the time of arrival of retinogeniculate afferents from the two eyes. For instance, in rodents, mammals with limited binocular vision, it is known that afferents from the contralateral eye precede those from the ipsilateral eye in invading the LGN (So et al., 1978; Bunt et al., 1981). On the other hand, virtually nothing is presently known about early events in the development of the retinogeniculate pathway in mammals with extensive binocular vision. Do the two sets of afferents arrive in concert, or does one set precede the other, as in rodents? Another, possibly related difference might be in the extent to which the two sets of afferents share the same territory within the LGN during development. In rodents, overlap is only partial, with afferents from the contralateral eye occupying more LGN territory than those from the ipsilateral eye at every stage of development. (So et al., 1978; Bunt et al., 1981). In primates, Rakic (1977a) has suggested that overlap may be complete initially, with input from both eyes equally spread throughout the LGN. Such differences in overlap could have a profound effect on the final pattern of afferent distribution within the nucleus.

It was with these issues in mind that the present study was made of the prenatal development of the cat's retinogeniculate pathway. The intention here is to provide, at the level of the light microscope, a description of the time course and pattern of development of connections in a mammal with extensive binocular vision. To do so, the methods of fetal surgery originally developed by Rakic (1976) for prenatal neuroanatomical studies in primates have been adapted to the cat. Fetuses of known gestational age between embryonic day 24 (E24) and birth (gestation in cats is 65 days) were given intraocular injections of ${ }^{3} \mathrm{H}$-amino acids or horseradish peroxidase (HRP), and then autoradiographic or histochemical methods were used to reveal the pattern of the retinogeniculate projection following orthograde axonal transport. Not surprisingly, in the development of the cat's retinogeniculate projection, as in that of other mammals (most notably the ferret), the segregated state is preceded by a period during which input from the two eyes is intermixed. However, as described below, the details of development differ in a number of important respects. A brief account of this work has appeared previously (Shatz and DeBerardino, 1980).

\section{Materials and Methods}

A total of 61 fetuses of known gestational age between E24 and E60, and 9 newborn kittens were studied. To obtain timed pregnancies, mature female cats housed in our breeding colony were placed with an experienced male for 24 to $48 \mathrm{hr}$ when they were observed to be in estrus by behavioral criteria (Scott, 1970). The time of mating was chosen as embryonic day 0 (E0), so that there is a maximum uncertainty of $48 \mathrm{hr}$ associated with each gestational age. As shown in Figure 1, a strict relationship exists between the gestational age estimated in this manner and the crown-rump length of each fetus included in this study, thereby verifying that pregnancies can be timed fairly accurately to within this $48-\mathrm{hr}$ window.

Fetal Surgery. Prior to surgery, each pregnant cat received intramuscular injections of atropine sulfatc $(0.05$ $\mathrm{mg} / \mathrm{kg}$ ), followed by a mixture of ketamine hydrochloride $(20 \mathrm{mg} / \mathrm{kg})$ and acepromazine $(0.2 \mathrm{mg} / \mathrm{kg})$ for induction of anesthesia. Five percent dextrose in Ringer's solution was infused intravenously for the duration of surgery. When necessary, uterine contractions were diminished with intravenous infusion of terbutaline sulfate (Brethine, $0.03 \mathrm{mg} / \mathrm{kg}$ ) or with the topical application of $5 \%$ cyclaine. An endotracheal tube was inserted so that anesthesia could be maintained with a combination of halothane $(0.5$ to $1.5 \%)$, nitrous oxide, and oxygen (1:2). Heart rate and expired $\mathrm{pCO}_{2}$ were routinely monitored.

The method of fetal surgery was modified slightly from that of Rakic (1976). To expose the two uterine horns, the skin and abdominal musculature were incised along the midline. In the cat, individual fetuses are easily identified as oval bulges along the uterine horns between E14 (when implantation occurs) and E35 (when the uterine horns appear uniformly smooth, externally) (Scott, 1970). Each fetus is "girdled" by a placenta iden-

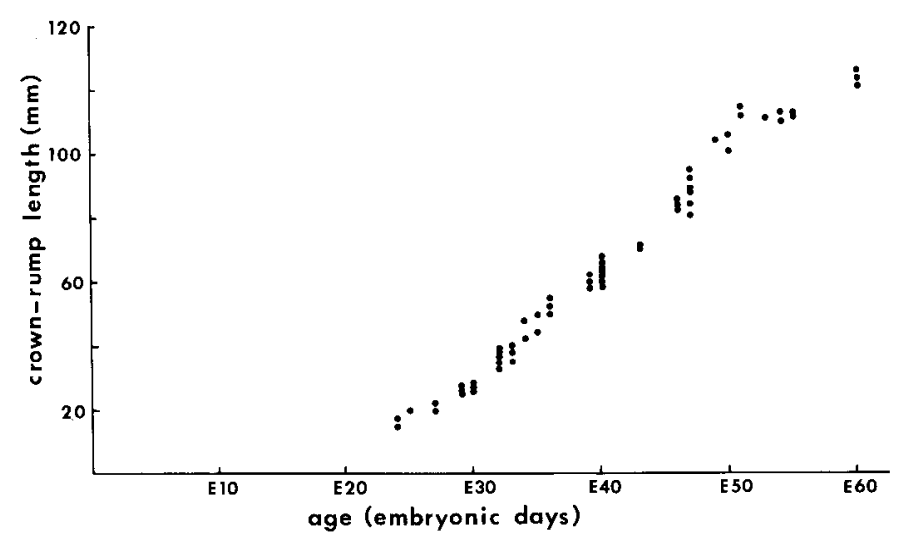

Figure 1. Graph of crown-rump length of each fetus measured immediately after perfusion versus embryonic age estimated from time of mating to show reliability of timing methods used here. 
tified by the extreme vascularization of the uterine wall. If the fetus is to survive the surgery, then, as in the monkey, care must be taken not to incise the placenta. In cases in which it was intended that some operated fetuses survive longer than others, or when a fetus was to be carried to term, fetuses destined to survive for the shortest time were operated closest to the cervix so that their removal would not cause expulsion of the remaining fetuses (McCance et al., 1966). Next the uterus and fetal membranes were incised, and the fetal membranes were sutured to the uterine wall to prevent loss of amniotic fluid. This procedure allows access to the fetus, which is partially exposed for the eye injections (see below). Once the injections had been made, the fetus was replaced inside the uterus, the uterine walls (including the fetal membranes), overlying muscle, and skin were sutured closed, and the mother cat was revived. After $24 \mathrm{hr}$ had elapsed to allow for axoplasmic transport, the mother cat was anesthetized as before, and the fetus was removed for perfusion and subsequent histological processing. Sterile surgical technique was employed for all procedures. Slightly greater than $80 \%$ (64 of 79 ) of all fetuses survived the surgical manipulations described here, with the majority of failures concentrated at the youngest ages.

Eye injections. Injections of ${ }^{3} \mathrm{H}$-amino acids and/or horseradish peroxidase (HRP) were made into the vitreous body of the eye by means of either a glass micropipette for fetuses E30 and younger, or a Unimetrics syringe with a 31 gauge needle for older fetuses and newborns. Puncture holes were sealed w. hh a drop of butyl cyanoacrylate glue (LO-835, Loctite Corp.) to prevent leakage of label. With this approach, controlled injections varying from 0.1 to $40 \mu \mathrm{l}$ can be made. Most animals were dually injected with HRP into one eye and ${ }^{3} \mathrm{H}$-amino acids into the other. For the HRP to be transported effectively in an anterograde fashion by retinal ganglion cells, a $40 \%$ solution (Boehringer-Mannheim type I) in $2 \%$ dimethylsulfoxide (DMSO) or 1\% lysophosphatidyl choline (Sigma type I) is required (Frank et al., 1980). Details of the injections made at different ages are given in Table I. Of the 73 animals listed, all survived the surgical procedures. However, in only $59 \%$ (43 of 73 ) of

TABLE I

Eye injection protocols

\begin{tabular}{|c|c|c|}
\hline Age at Perfusion & $\begin{array}{l}\text { Number } \\
\text { Studied }\end{array}$ & Amount Injected \\
\hline E24 to E30 & 12 & $\begin{array}{l}0.1-0.5 \mu \mathrm{l} 40 \% \text { HRP } \\
0.1-0.5 \mu \mathrm{l}(10-50 \mu \mathrm{CI}){ }^{3} \mathrm{H} \text {-amino } \\
\quad \text { acids }\end{array}$ \\
\hline $\mathrm{F} 32$ to $\mathrm{E} 40$ & 25 & $\begin{array}{l}1-3 \mu \mathrm{l} 40 \% \mathrm{HRP} \\
0.5-3 \mu \mathrm{l}(50-200 \mu \mathrm{CI}){ }^{3} \mathrm{H} \text {-amino } \\
\quad \text { acids }\end{array}$ \\
\hline $\mathrm{E} 43$ to $\mathrm{E} 50$ & 14 & $\begin{array}{l}5-20 \mu \mathrm{l} 40 \% \mathrm{HRP} \\
5-10 \mu \mathrm{l}(200-250 \mu \mathrm{Ci}){ }^{3} \mathrm{H} \text {-amino } \\
\quad \text { acids }\end{array}$ \\
\hline $\mathrm{E} 51$ to $\mathrm{E} 60$ & 13 & $\begin{array}{l}20-30 \mu l 40 \% \mathrm{HRP} \\
10-20 \mu \mathrm{l}(250-500 \mu \mathrm{Ci}){ }^{3} \mathrm{H} \text {-amino } \\
\quad \text { acids }\end{array}$ \\
\hline P1 to P3 & 9 & $\begin{array}{l}40 \mu \mathrm{l} 40 \% \mathrm{HRP} \\
25 \mu \mathrm{l}(500 \mu \mathrm{Ci}-\mathrm{I} \mathrm{mCi}){ }^{3} \mathrm{H} \text {-amino } \\
\quad \text { acids }\end{array}$ \\
\hline
\end{tabular}

the cases did one or both eye injections succeed, as judged by a consistent and reproducible pattern of labeling within the LGN and the complete, homogeneous labeling of the contralateral superior colliculus (Williams and Chalupa, 1982). Failures could be attributed to retinal damage during the eye injections.

In initial experiments, $\left[{ }^{3} \mathrm{H}\right]$ proline $(20$ to $40 \mathrm{Ci} / \mathrm{mm})$ was selected for use in eye injections since it has been shown to be transported in the fetal visual system (Rakic, 1977a). However, it is now known that, at least in the postnatal cat's LGN, following an intraocular injection of $\left[{ }^{3} \mathrm{H}\right]$ proline some of the radioactive label transported to the IGN may spill over from the retinal ganglion cell terminals and diffuse away; further, the amount of this "spillover" is greater the younger the animal (LeVay et al., 1978). There was, therefore, the worry that during fetal development spillover could obscure the precise pattern of retinal input, particularly with the use of long postinjection survival times. That this was indeed the case became clear when autoradiographs from two E46 fetuses, one injected $I$ week earlier and the other injected $24 \mathrm{hr}$ earlier, were compared. Consequently, in an effort to minimize spillover, survival times of $24 \mathrm{hr}$ or less have been used routinely here.

Even with 24-hr survival times, spillover probably contributes somewhat to the pattern of autoridiographic labeling. This was indicated by another control experiment in which, at E50, the same eye was injected with both HRP and $\left[{ }^{3} \mathrm{H}\right]$ proline and the LGN labeling pattern produced $24 \mathrm{hr}$ later by the two tracers was compared. As expected, the two patterns, histochemical and autoradiographic, were highly similar. However, particularly on the side contralateral to the eye injection, regions labeled autoradiographically were consistently more extensive than those labeled histochemically, indicating either that radioactive label had spilled over from labeled terminals or, equally possible, that it is a slightly more sensitive marker than the HRP reaction product. In an additional effort to minimize spillover, therefore, $\left[{ }^{3} \mathrm{H}\right]$ leucine ( 40 to $60 \mathrm{Ci} / \mathrm{mm}$ ) was used in the majority of eye injections. This amino acid is transported equally well and, at least in adults, is thought to not be transneuronally transported to the same degree as proline.

Histology. Vascular (intracardiac) perfusion of even the smallest embryos (E24) was accomplished by means of a peristaltic pump attached to needles of different gauge (or a glass micropipette) depending on fetal age. Animals were perfused initially with $0.1 \mathrm{M}$ phosphate buffer $(\mathrm{pH} 7.3)$. For dually injected animals this was followed by a mixture of $1 \%$ paraformaldchyde, $1.25 \%$ glutaraldehyde, and 5\% sucrose in the same buffer. In instances where autoradiography alone was intended, $4 \%$ paraformaldehyde $/ 5 \%$ sucrose in $0.1 \mathrm{~m}$ phosphate buffer was used. In one series of animals, brains were blocked in the coronal plane; in another, they were blocked horizontally. Tissue was left to harden at $4^{\circ} \mathrm{C}$ overnight in the same fixative. Material to be frozen-sectioned was cryoprotected by the further addition of $20 \%$ sucrose to the fixative.

For HRP histochemistry, brains were embedded in a rapidly hardening gelatin/albumin mixture and sectioned at $50 \mu \mathrm{m}$ either on a freezing microtome or a Vibratome. (Vibratome sectioning avoided the introduction of freez- 
ing artifacts, especially troublesome in younger fetuses.) Sections were reacted according to the tetramethylbenzidine (TMB) method of Mesulam (1976). Here, the method was modified slightly to minimize tissue shrinkage and crystal formation. Sections were rinsed in $0.2 \mathrm{M}$ sodium acetate buffer ( $\mathrm{pH} \mathrm{6)}$, soaked for $10 \mathrm{~min}$ in $2 \%$ DMSO in the same buffer, rinsed briefly, and then incubated and reacted according to the method of Mesulam (1976) except that the $0.2 \mathrm{M}$ sodium acetate buffer $(\mathrm{pH} 6)$ was substituted for Mesulam's pH 3.3 buffer, and the quantity of sodium nitroprusside was reduced to $75 \mathrm{mg} /$ $100 \mathrm{ml}$. Reacted sections were then rinsed and mounted from a $0.14 \mathrm{~m}$ sodium acetate buffer, $\mathrm{pH}$ 3.3. Alternate sections were counterstained with neutral red. Further details of these modifications will be presented elsewhere (C. J. Shatz and M. Siegel, manuscript in preparation).

For autoradiography, sections 20 or $50 \mu \mathrm{m}$ thick (dually injected animals) were dipped in Kodak NTB-2 emulsion, exposed for 1 to 2 weeks, and developed in D-19. In dually injected cases, sections immediately adjacent to those reacted for HRP histochemistry were chosen, since it is not possible to dip the reacted sections directly (the TMB reaction product is bleached by developer). After development, sections were lightly counterstained with cresyl violet. Frequently, another complete set of undipped, unreacted sections was cresyl violet stained deeply to reveal cytoarchitectonic details. The distribution of radioactive label and HRP reaction product was viewed in bright- and dark-field illumination and recorded by means of photographs and camera lucida drawings.

Computer measurements of overlap. In order to obtain a more quantitative description of the time course and pattern of segregation, two methods were used to estimate the extent to which afferents from the two eyes shared the same territory (overlapped) within the LGN at different ages. At each age selected, LGN regions occupied by radioactive label and/or HRP reaction product ipsilateral and contralateral to each eye injection were examined in horizontal section with dark-field optics and then drawn with the aid of the camera lucida. It must be emphasized that only in horizontal (but not coronal) sections is it possible at each age to reliably identify and draw the dorsal LGN exclusive of its immediate neighbors: the ventral LGN, medial interlaminar nucleus (MIN), perigeniculate nucleus, and the optic tract. In view of the variability inherent in the anterograde labeling methods used here (due to variations in quantities of injected label, in exposure times, and in intensity of the HRP reaction product), a rigid quantification of labeling density was not attempted. The border between labeled and unlabeled regions was determined by eye. Regions containing very sparse and scattered label were designated as unlabeled for the purposes of these measurements.

The first method was to measure directly the amount of overlap in a series of double-labeled animals aged E32, $\mathrm{E} 40, \mathrm{E} 47, \mathrm{E} 54, \mathrm{E} 60$, and postnatal day $2(\mathrm{P} 2)$ in which both eye injections had succeeded (see, for example, Fig. 13). At each age, two adjacent sections through the midportion of the LGN were processed: one for autoradiography and the other for HRP histochemistry. A camera lucida drawing was made first of the distribution of HRP reaction product within both LGNs. (Again note that label within the optic tracts was excluded.) Next the autoradiographic labeling pattern was drawn superimposed by approximately adjusting the camera lucida magnification to compensate for differential shrinkage of the tissue. The cross-sectional areas occupied by the resulting dually labeled regions were measured directly with the aid of a digitizing tablet connected to an Apple computer and expressed as a percentage of the total cross-sectional area of the LGN in the same drawing, thereby providing a measure of overlap: the percentage of area of dual innervation at each age. For each determination of total LGN cross-sectional area, the borders of the LGN were located histologically from the brightfield view of the same sections counterstained with cresyl violet or neutral red. At the earliest ages (E40 and younger), where histological identification is more difficult, LGN borders were taken to be coincident with the labeling pattern contralateral to the eye injection for reasons discussed under "Results."

In a second more indirect method (Fig. 14 and 15), the area occupied by a single label within the ipsilateral and contralateral LGNs was measured in a larger series of fetuses aged E32 (2 animals), E35 (1 animal), E40 (3 animals), E47 (3 animals), E54 (1 animal), E60 (2 animals), and ( $\mathrm{P} 1$ (1 animal), P2 (3 animals), and adult (1 animal). In each case, camera lucida drawings were made of six to eight sections selected from the central twothirds of the LGN. In instances where both eye injections had succeeded, two separate sets of drawings were made, one for each label. Again, with the aid of a digitizing tablet, the cross-sectional areas occupied by label ipsilateral or contralateral to the eye injection were computed from the drawings and totaled for all six to eight sections. Each total was next expressed as a percentage of the total cross-sectional area of the nucleus ipsilateral or contralateral to the eye injection in the same sections as described above. Overlap, the percentage of territory shared by both eyes at a particular age was then expressed as: [\% area occupied by label on ipsilateral side plus $\%$ area labeled on contralateral side] $-100 \%$.

\section{Results}

Time course and pattern of development of the retinogeniculate pathway. By birth, the segregation of retinal afferents within the lateral geniculate nucleus appears quite adult-like when viewed in coronal section. This is shown in Figure $2 A$, an autoradiograph photographed in dark-field of the LGNs from a 1-day-old kitten (P1) that had received an intraocular injection of $\left[{ }^{3} \mathrm{H}\right]$ leucine 24 hr previously. Regions containing radioactive label are sharply delimited, and it is possible to identify unambiguously each LGN layer (A, A1, C, C1, and C2) (Guillery, 1970) on the basis of the characteristic labeling pattern (Hickey and Guillery, 1973). In spite of this clarity in the pattern of the retinogeniculate projection, the subdivision of the nucleus into its several distinct cell laminae and acellular intralaminar regions is much less evident, as shown in Figure $2 B$, a cresyl violet-stained section adjacent to that of Figure $2 A$.

Embryonic day 24 was selected as an appropriate fetal age at which to begin this developmental study of the retinogeniculate pathway. Windle (1935) had reported 

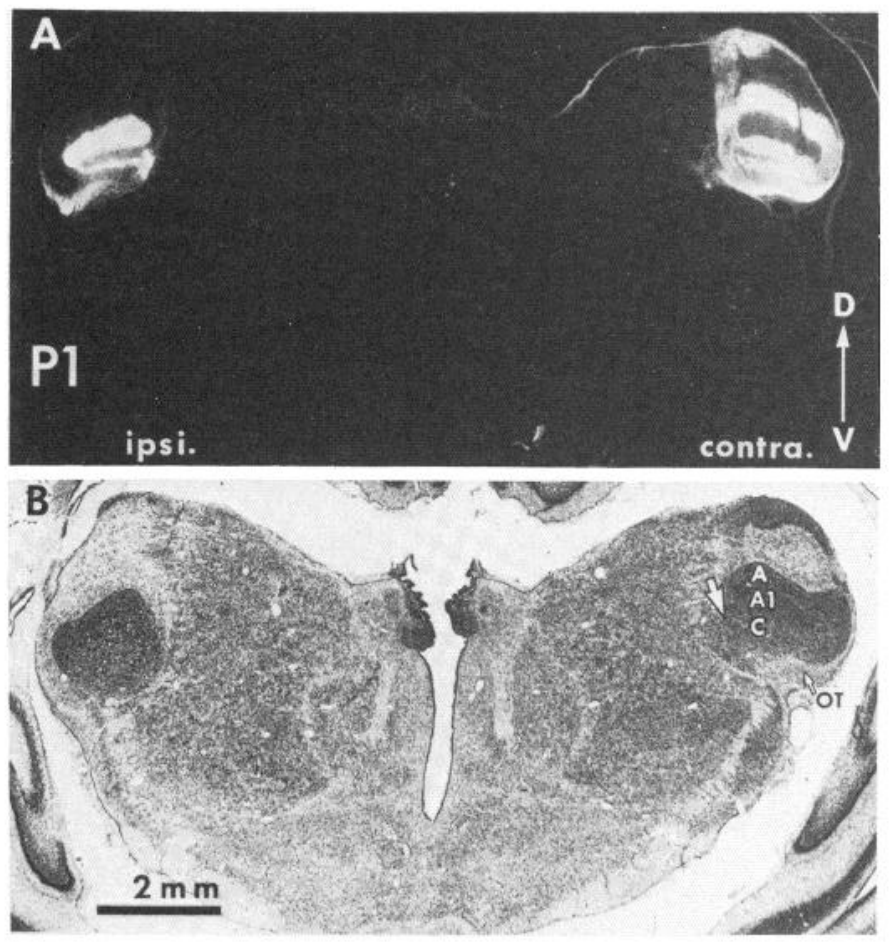

Figure 2. The retinogeniculate projection pattern as seen in coronal section at postnatal day $1(P 1)$ contralateral (right) and ipsilateral (left) to an intraocular injection of $\left[{ }^{3} \mathrm{H}\right]$ leucine. $A$, Autoradiograph in dark-field, to show the distribution of radioactive label contained within the afferents (white regions) in both LGNs. $B$, Adjacent cresyl violet-stained section to show the histological picture at P1. LGN cell laminae are indicated on the right: $A, A 1$, and $C$. $C$ refers collectively to all three $\mathrm{C}$ laminae: $\mathrm{C}, \mathrm{C} 1$, and $\mathrm{C} 2$. The large white arrow indicates the position of the medial interlaminar nucleus (MIN). OT optic tract. Sections are oriented dorsal $(D)$ up and ventral $(V)$ down. Calibration bar, $2 \mathrm{~mm}$ for both $A$ and $B$.

that at this age the optic nerve can be identified by means of silver staining, as is verified here by simple inspection of Figure 3 (arrow), a cresyl violet-stained coronal section through the entire head of an E24 fetus. At this age, however, the LGN itself is nonexistent; this is shown in Figure $4 A$, a coronal section taken further posteriorly, through the diencephalon of the same E24 fetus. The dorsolateral region, the future site of the LGN (Fig. $4 A$, arrows), consists of a primitive, darkly stained ventricular zone, which, in the higher power view of Figure $4 B$, is encased only by a thin intermediate zone and is capped externally by a cell-free marginal zone. The histological picture at E24 fits well with recent $\left[{ }^{3} \mathrm{H}\right]$ thymidine studies of LGN neurogenesis which have revealed that neurons comprising the cat's LGN are generated at the ventricular zone during the 1-week period beginning on E24 (Hickey and Cox, 1979; Shatz, 1981). In this context then, the clear thickening of the intermediate zone subjacent to the marginal zone visible 3 days later at E27 (Fig. 4, $C$ and $D$ ) can be interpreted as consisting of, at least in part, postmitotic neurons belonging to the nascent LGN.

It is not until 5 days later, at E32, that retinal afferents can be unambiguously identified within the anlage of the LGN by the anterograde transport methods used here.
For instance, Figure 5 shows, in the coronal plane, a series of dark-field autoradiographs (left) and adjacent cresyl violet-stained sections (right) of the diencephalon from three fetuses aged E32, E36, and E39 that had received intraocular injections of ${ }^{3} \mathrm{H}$-amino acids $24 \mathrm{hr}$ previously. At E32 (Fig. 5, $A$ and $B$ ) the optic tracts (ot) form thin, heavily labeled bands along the outermost rim of the diencephalon, but only on the contralateral (right) side can label be seen extending medial to the tract, actually within the LGN anlage (compare Fig. 5, $A$ and $B$ ). Prior to E32, label is confined almost exclusively to the optic tract both ipsilaterally and contralaterally, with labeling of the contralateral tract consistently greater than that of the ipsilateral tract as early as the eye injections have succeeded: at E28. (A thorough consideration of results from eye injections made prior to E32 is in preparation. See also Shatz and Kliot, 1982.)

Histologically, the period between E32 and E39 is characterized by the gradual transformation of the LGN anlage from a barely identifiable thin sheet of cells perched on the dorsolateral margin of the thalamus at E32 (Fig. $5 B$, arrows) to a distinctive teardrop-shaped cellular mass clearly delineated from more medial thalamic structures by E39 (Fig. $5 F$, arrows). The transformation is due, in part, to the progressive arrival of many late-generated LGN neurons following their migration from the ventricular zone (Shatz, 1981) and to the concurrent invasion of the LGN by retinogeniculate afferents from both contralateral and ipsilateral eyes, which is quite evident by E39 (Fig. $5 E$ ). Note, however, that the

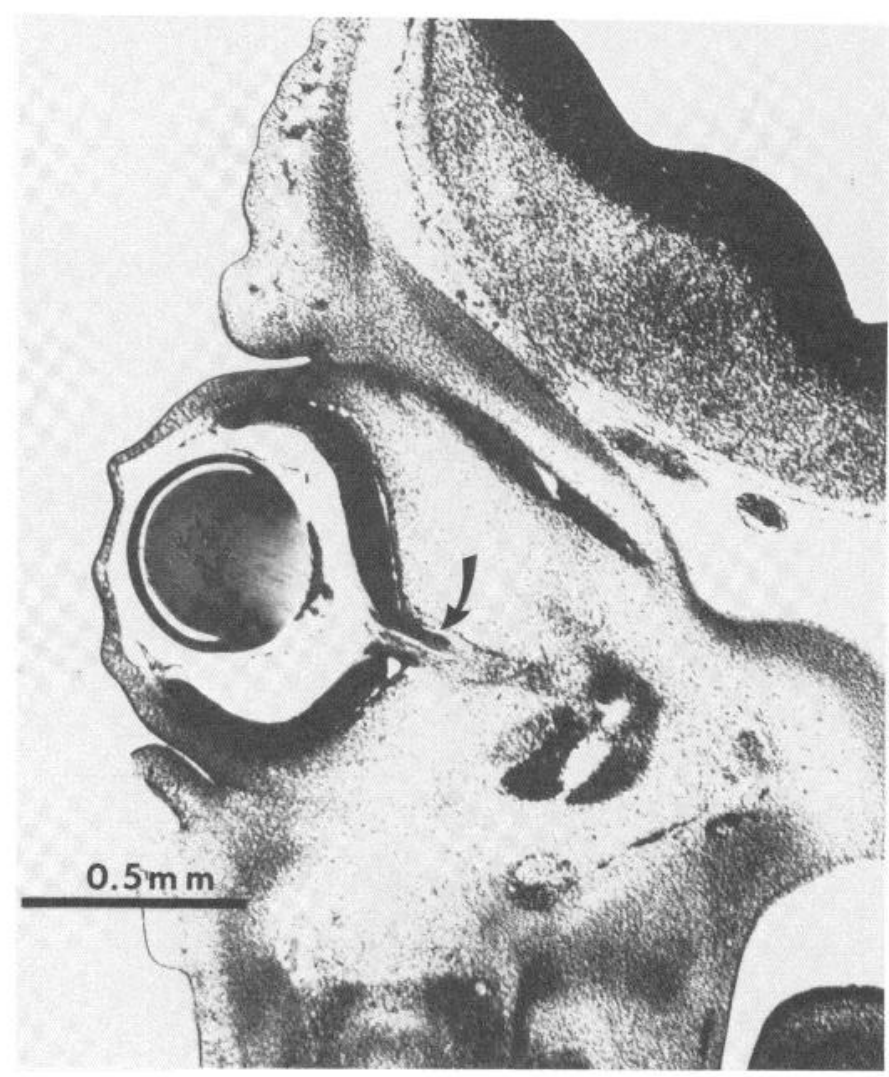

Figure 3. A cresyl violet-stained coronal section through the entire head of an E24 fetus to illustrate that the optic nerve (arrow) is already present by this age. 

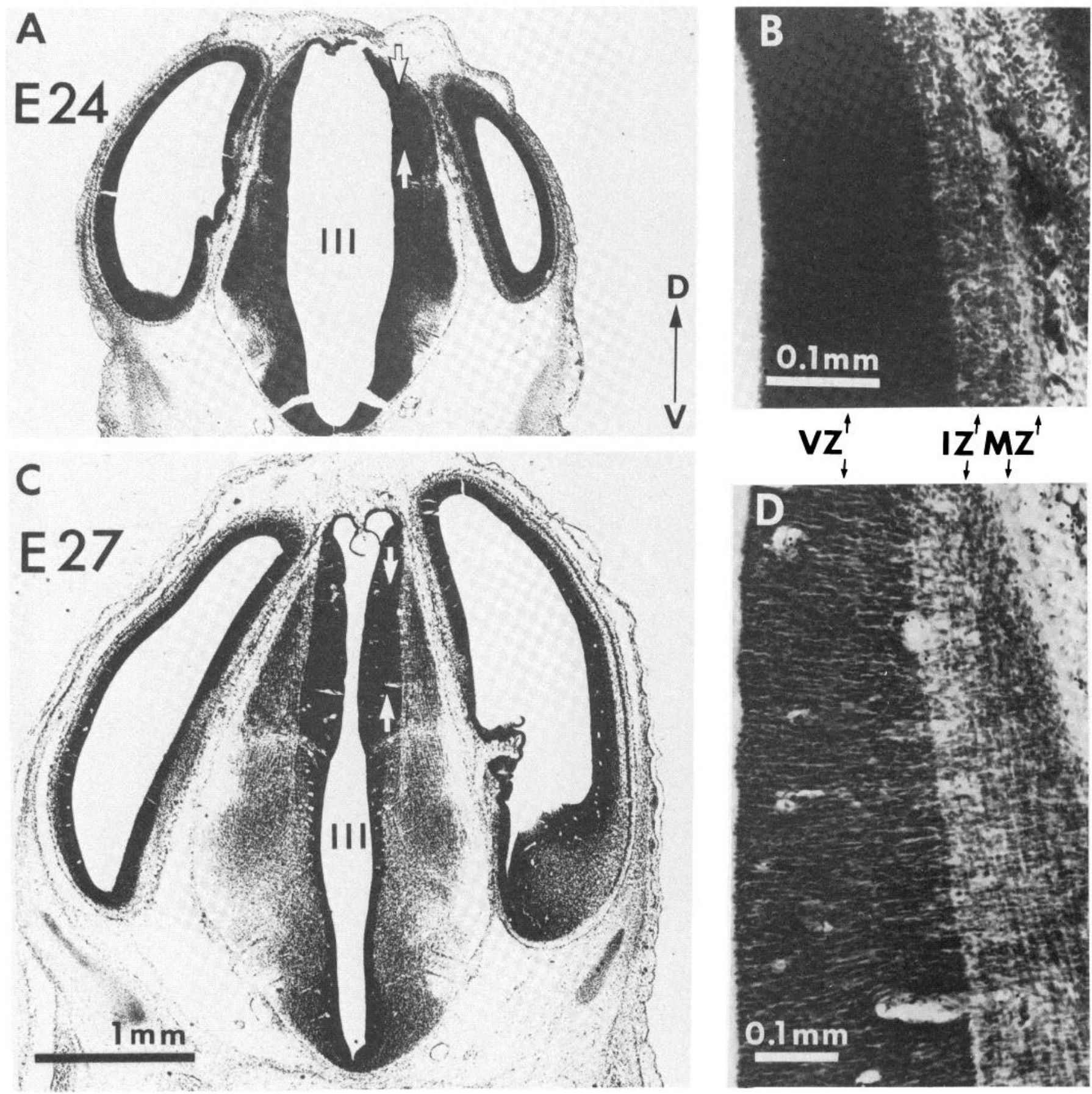

Figure 4. Cresyl violet-stained coronal sections through the heads of two fetuses aged E24 $(A$ and $B)$ and E27 $(C$ and $D)$ to show the primitive histological appearance of the diencephalon at these ages. In $A$ and $C$, the diencephalon is flanked on either side by the telencephalic vesicles. $I I I$, third ventricle. The calibration bar $(1 \mathrm{~mm})$ in $C$ also refers to $A$. The approximate location of the dorsal thalamic region containing future LGN is indicated between each pair of white arrows in $A$ and $C$. The same arrows delineate the area included in high power views of $B$ and $D$. Note that at E24 $(B)$, the thalamus consists largely of a ventricular zone $(V Z)$ and a marginal zone $(M Z)$, while by E27 $(D)$ a pronounced intermediate zone $(I Z)$ has appeared. In $A, D$ is dorsal; $V$ is ventral.

afferents, particularly on the ipsilateral side, are not distributed throughout the entire mediolateral extent of the LGN anlage as defined histologically. In Figure $5 E$, radioactive label is confined to lateral regions of the LGN, well away from the white arrows that mark the medial border of the LGN as ascertained from subse- quently staining the autoradiograph with cresyl violet (compare also Fig. $5 F$ ).

Coincidence between the autoradiographic labeling pattern and the boundaries of the LGN as determined histologically is seen by E46, as shown in Figure 6, $A$ and $B$. At this age, however, distinct gaps appear in the 

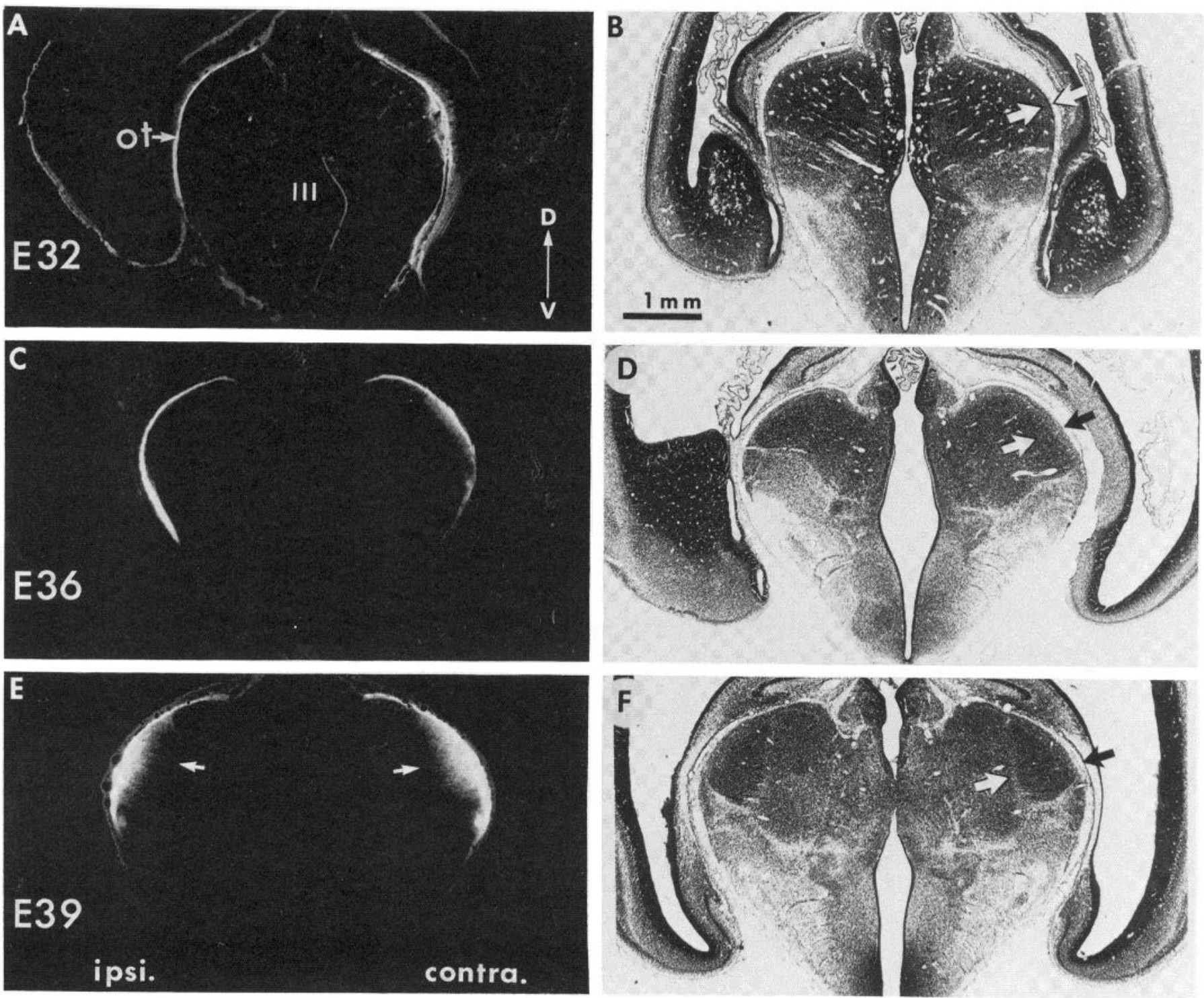

Figure 5. The retinogeniculate projection pattern as viewed in the coronal plane in three progressively older fetuses aged E32 $(A$ and $B)$, E36 $(C$ and $D)$, and E39 $(E$ and $F)$ that had each received an intraocular injection of ${ }^{3} \mathrm{H}$-amino acids. At each age, on the left $(A, C, E)$ is an autoradiograph, shown in dark-field, of a section through the diencephalon to illustrate the distribution of labeled afferents (seen in white) within the optic tract (ot) and LGNs ipsilateral (left) and contralateral (right) to the eye injection. On the right $(B, D, F)$ are the adjacent cresyl violet-stained sections. In $B, D$, and $F$, the approximate location of the LGN is indicated between each pair of arrows (right-hand side of section). Note that at E39, the medial border of the LGN as determined histologically ( $E$ and $F$, white arrows) does not coincide with the afferent labeling pattern. At this age, the ventral lateral geniculate nucleus $(F$, cell mass immediately below the black area) and the retinal projection to it $(E$, most ventral and lateral label) can also be seen clearly. $1 \mathrm{~mm}$ the calibration bar in refers to $A$ to $F$. Dorsal $(D)$ is up and ventral $(V)$ is down. $I I I$, third ventricle.

retinogeniculate projection, both ipsilaterally and contralaterally. These gaps become progressively more pronounced during the next 2 weeks (E54: Fig. $6, C$ and $D$; E60: Fig. $6, E$ and $F$ ), suggesting that afferents from the two eyes are in the process of segregating from each other between E46 and E60. However, unambiguous identification of the future LGN layers, either from the anterograde labeling patterns or from an inspection of the histology in cresyl violet-stained sections, was not possible.

The explanation for this difficulty is that the coronal plane of section, although it is the plane of choice for viewing the layers in the adult, is not optimal during prenatal life due to a progressive change in position of the cat's LGN during development. Therefore, another series of fetuses was studied, but this time, sections were taken in the horizontal plane. Even at P2, horizontal sections revealed clearly the subdivision of the afferent input from the retina into the appropriate $\mathrm{A}$ and $\mathrm{C}$ layers, as shown in the photographs of Figure 7, $A$ and $B$. A comparison of Figure 7 with Figure 2 (coronal) also indicates that the cytoarchitectural subdivision of the nucleus into laminae can be more clearly discerned in horizontal sections. Thus, at birth, the layers of the cat's LGN run as a series of parallel sheets roughly orthogonal to the horizontal plane. This observation is in perfect 

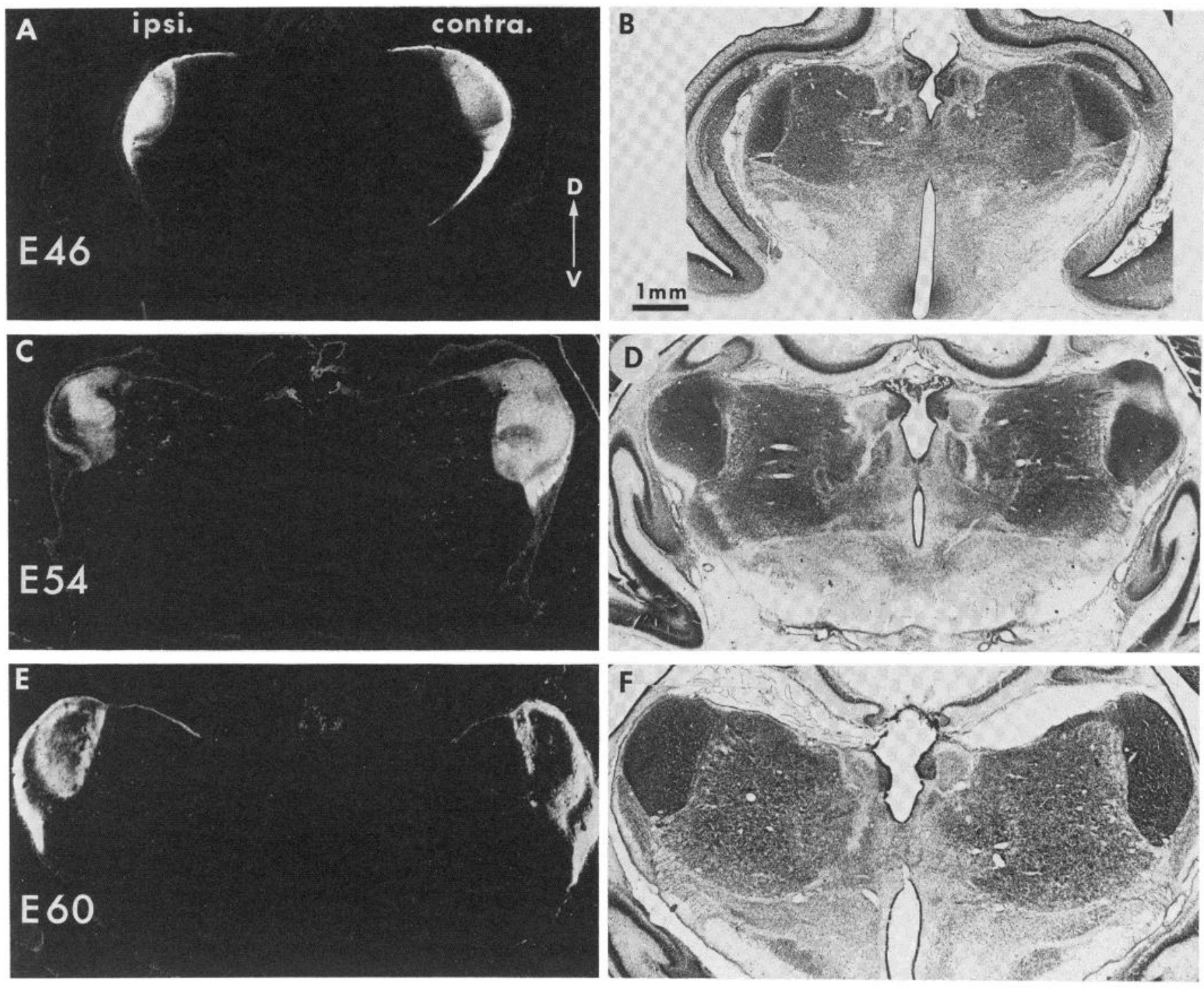

Figure 6. Coronal sections through the diencephalon illustrating the pattern of the retinogeniculate projection in a series of three older fetuses aged E46 $(A$ and $B)$, E54 $(C$ and $D)$, and E60 ( $E$ and $F$ ). On the left are dark-field photographs of the autoradiographic $(A)$ or peroxidase $(C$ and $E$ ) labeling patterns ipsilateral (left) and contralateral (right) to an intraocular injection of ${ }^{3} \mathrm{H}$-amino acids $(A)$ or $\operatorname{HRP}(C$ and $E)$. On the right are the adjacent cresyl violet-stained sections. The calibration bar $(1 \mathrm{~mm})$ in $B$ refers to $A$ to $F$.

accord with those of Kalil (1978), who thoroughly documented the progressive $90^{\circ}$ displacement of the nucleus in the sagittal plane that occurs during the first 4 postnatal months in the cat, thereby shifting the layers into the adult configuration in which they run as sheets mostly parallel to the horizontal plane.

When horizontal sections are taken through the thalamus of an E60 fetus, the familiar laminar arrangement of afferent input to the LGN is revealed clearly, as shown in the dark-field autoradiograph of Figure $8 A$. For instance, ipsilateral to the eye injection, the location of layers $\mathrm{A} 1$ and $\mathrm{C} 1$ can be inferred unambigiously from the pattern of labeling which, at this age, is remarkably adult-like (see below and Figs. 13 to 15 for further substantiation). Despite this relative maturity in the pattern of retinal input, there is only a faint hint of an interlaminar region between laminae $\mathrm{A}$ and $\mathrm{A} 1$ at $\mathrm{E} 60$, as can be seen from inspecting the adjacent cresyl violet-stained section in Figure $8 B$, and from examining another nearby section at higher power (Fig. 9).

Even 1 week earlier, at E54, correlates of the adult retinogeniculate projection can be recognized in horizontal section, as shown in the dark-field photograph of Figure $8 C$. For instance, afferent input to future layers A and A1 is easily identified (compare with Fig. 7) and the border between the contralateral input to $\mathrm{A}$ and the ipsilateral input to $\mathrm{A} 1$ is quite sharp. On the other hand, although the general location of the $\mathrm{C}$ layers can be determined here, the pattern of labeling is much less distinct, making the precise subdivision of the $\mathrm{C}$ layers into $\mathrm{C}, \mathrm{C} 1$, and $\mathrm{C} 2$ a difficult task. The task becomes even more difficult at E47, as shown in the darkfield photograph of Figure $8 E$. At this age, the pattern of the retinogeniculate projection bears little resemblance to 

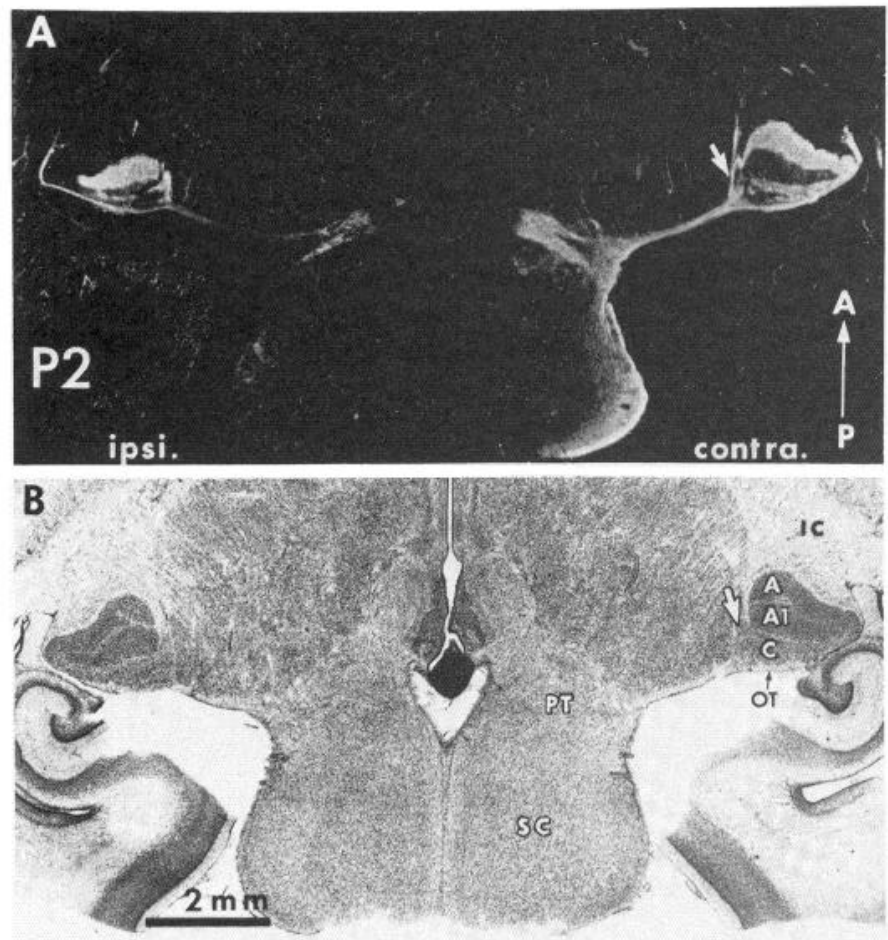

Figure 7. The retinogeniculate projection at postnatal day 2 $(P 2)$, viewed in the horizontal plane. $A$, Dark-field photograph of the afferent labeling pattern ipsilateral (left) and contralateral (right) to an intraocular injection of HRP. This horizontal section (and others to follow) passes through the dorsal thalamus (top) and midbrain (bottom), revealing the retinal projections to the LGN, MIN (right, white arrow), pretectum, and superior colliculus. $B$, Adjacent cresyl violet-stained section to show the pattern of cellular lamination within the LGN. On the right, laminae $A, A 1$, and the $C$ laminae are indicated. ( $C$ refers to all three $\mathrm{C}$ laminae; $\mathrm{C}, \mathrm{C} 1$, and $\mathrm{C} 2$.) The white arrow indicates the location of the MIN. $I C$, internal capsule; $O T$, optic tract; $P T$, pretectum; $S C$, superior colliculus. The calibration bar $(2 \mathrm{~mm})$ is for $A$ and $B$. Note that these and all other horizontal sections are oriented so that anterior $(A)$ is up and posterior $(P)$ is down. (Section orientations are indicated in $A$.)

that seen in the adult or at P2. Nevertheless, it is possible to make a number of inferences about the position of the future A laminae here. Note first that the gap present in labeling at the posterior pole of the nucleus on the contralateral side (Fig. $8 E$, right) is only partially complemented by an elongated patch of label on the ipsilateral side (Fig. $8 E$, left). Taken together, the labeling pattern on both sides suggests that the ipsilateral label represents the afferent input largely to future layer $\mathrm{A} 1$ and that this ipsilateral input is overlapped by input from the contralateral eye everywhere except at the posterior pole of the nucleus where segregation is just beginning (see also Figs. 13 to 15). If so, then by analogy with the adult and older embryos (Figs. 7 and 8), the region anterior to the gap and bounded medially by the white matter of the internal capsule (see Fig. $8 F$ ) is destined to become layer $\mathrm{A}$, while that posterior to the gap and bounded laterally by the optic tract will become the future $\mathrm{C}$ layers. These suggestions are also supported by the results of $\left[{ }^{3} \mathrm{H}\right]$ thymidine studies of LGN neurogenesis (see "Discussion" and Shatz, 1981).
At E40 and earlier (E35 and E32), the pattern of the retinogeniculate projection is considerably simplified and consists of thin continuous bands of label that run along the anterolateral margin of the thalamus both ipsilaterally and contralaterally (Fig. 10). Contralaterally, however, the extent of label is consistently greater than that present ipsilaterally at each age. This inequality is most evident at E32 (Fig. 10E), when label on the ipsilateral side is restricted to the optic tract while that on the contralateral side is found not only within the tract, but also more medially, embedded within the LGN anlage. Figure 11, a high power dark-field photograph of the autoradiograph of Figure $10 E$, shows this organization most clearly, thereby confirming the proposal made earlier from the results of the coronal series, that afferents from the contralateral eye precede by several days those from the ipsilateral eye in invading the LGN. Despite the irregularity between the two sides of the thalamus, the fact that label is present within corresponding territories suggests that by E40 there is overlap of input from the two eyes, particularly at the lateral margin of the LGN, a suggestion supported directly by the double-label experiments described below.

Inspection of the anterograde labeling patterns seen in horizontal section between E32 and E40 gives no indication of the location of future LGN layers. Nevertheless, it does aid in the histological identification of the nucleus itself, as seen in the cresyl violet-stained sections of Figure 10. Prior to E40, the LGN anlage can be identified as the thin sheet of cells perched on the anterolateral margin of the dorsal thalamus and separated from more medial thalamic structures by a faint acellular gap (Fig. $10, D$ and $F$, white arrows). At $\mathrm{E} 40$, the labeling pattern indicates that the LGN anlage consists at minimum of a comma-shaped nuclear mass situated along the lateral margin of the thalamus. Histologically, however, the nucleus could also extend further medially to include the area up to the white arrow on the right-hand side of Figure $10 B$. This ambiguity in the location of the medial border of the nucleus at this age can be explained by the finding, based on $\left[{ }^{3} \mathrm{H}\right]$ thymidine autoradiography, that the more medial region at E40 consists of late-generated neurons still migrating en route to the LGN (Shatz, 1981).

In comparing development of the retinogeniculate pathway as viewed in the coronal or the horizontal plane, it is clear that results from the two studies are complementary. By E32, retinal afferents from the contralateral eye invade the anlage of the LGN, followed by those from the ipsilateral eye shortly thereafter (E35). Then, during the subsequent 5-week period, both sets of afferents first overlap and next segregate from each other to assume the adult-like laminar pattern of afferent input by birth. As seen above, several important details of this process are revealed with great clarity in the horizontal but not the coronal plane. Perhaps most remarkable of all is the progressive displacement of the entire nucleus during prenatal life evident in the horizontal sections of Figures 8 and 10. For instance, a simple inspection of the position of the LGN in Figure 8 shows that what is medial at E60 (Fig. 8, $A$ and $B$ ) corresponds to what was posterior at E47 (Fig. 8, $E$ and $F$ ); what is lateral at E60 was anterior at E47. The sequential transposition of the LGN is illustrated in its entirety in Figure 12, a series 

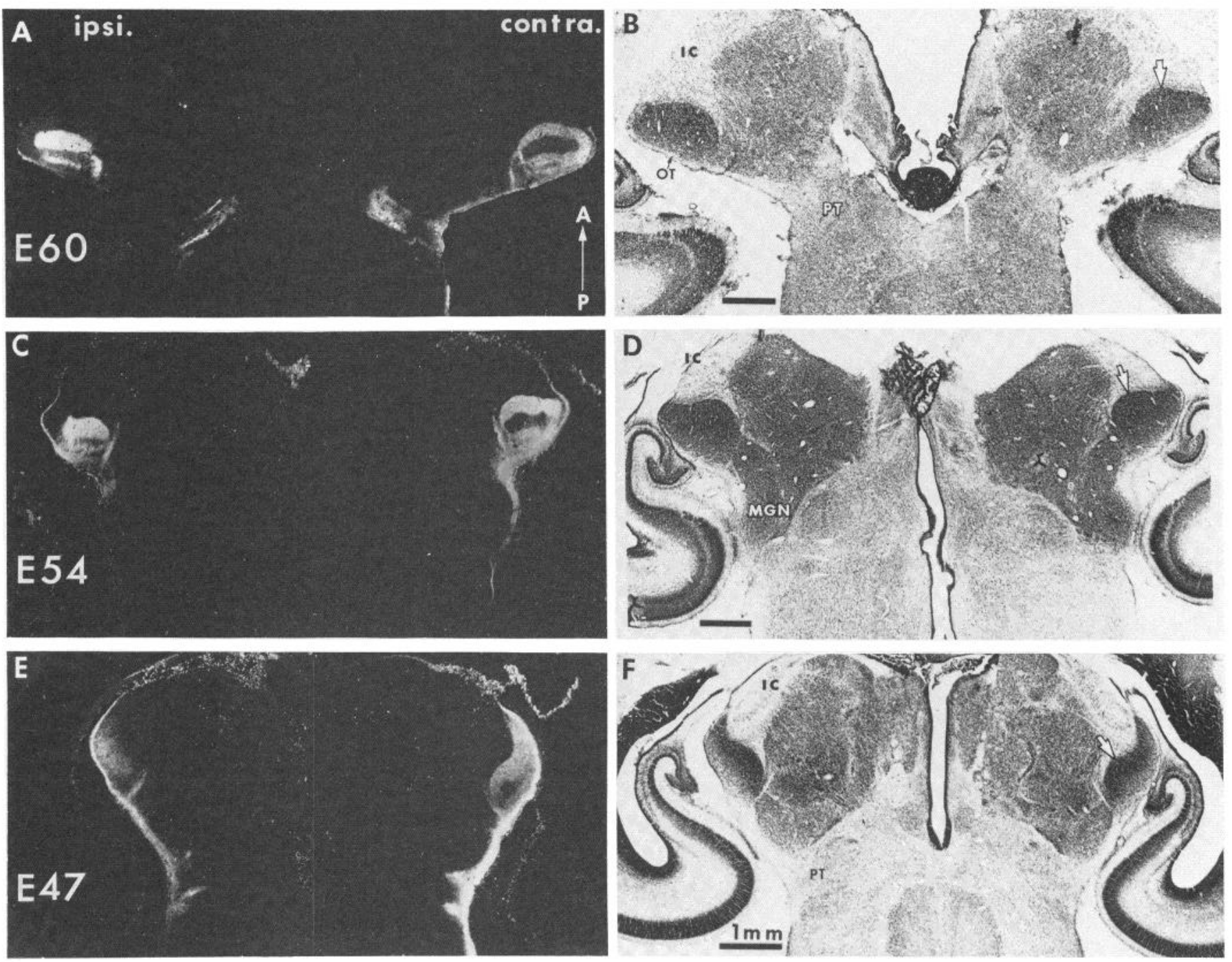

Figure 8. Horizontal sections through the dorsal thalamus and midbrain of three fetuses aged E60 $(A$ and $B)$, E54 $(C$ and $D)$, and $\mathrm{E} 47(E$ and $F)$ to illustrate the pattern of the retinogeniculate projection at progressively younger ages. On the left are darkfield photographs of the labeled afferents ipsilateral (left) and contralateral (right) to an intraocular injection of ${ }^{3} \mathrm{H}$-amino acids $(A)$ or HRP $(C$ and $E$ ). At each age, the retinal projections to the LGN and MIN are visible; at E60 and E47, that to the pretectum is also evident due to the plane of section. On the right are the adjacent cresyl violet-stained sections. The white arrow in each photograph $(B, D, F$ : right LGN) indicates the inner border of the LGN immediately apposing the internal capsule (IC). In $B$ and $D$, note the lack of cellular lamination within the nucleus despite the relative maturity in the pattern of the afferent input seen in $A$ and $C$. OT, optic tract; $M G N$, medial geniculate nucleus; $P T$, pretectum. The calibration bar (1 mm) in $F$ is for $A$ to $F$.

of camera-lucida drawings of horizontal sections similar to those of Figures 8 and 10. Between E40 and E60, the nucleus is displaced roughly $90^{\circ}$ laterally and posteriorward in the horizontal plane to acquire the mediolateral axial alignment typical of the adult. Prior to this displacement, the medial geniculate nucleus (Fig. 12, E40: $\mathrm{mgn}$ ) is actually situated lateral to the LGN.

Segregation of the retinogeniculate afferents. From the pictorial study presented above it has been possible to infer that in the development of retinogeniculate connections, afferents from the two eyes first overlap and then undergo a process of segregation within the LGN similar in broad outline to that seen in other mammals. In order to obtain a less impressionistic description of this process and its time course in the cat, two more quantitative approaches were employed.
In the first, the amount of overlap between the two sets of afferents was assessed directly in a series of double-labeled animals that had each received an injection of HRP in one eye and $\left[{ }^{3} \mathrm{H}\right] l$ leucine in the other. Superposition of the autoradiographic and peroxidase labeling patterns contained within two adjacent horizontal sections by means of camera lucida drawings (see "Materials and Methods") made it possible to determine the location and extent of dually labeled regions within the dorsal LGN at each age. Results using this approach are shown in Figure 13, drawings from a series of six animals to illustrate the distribution of the two labels. At each age, the left-hand LGN was contralateral to the eye receiving the $\left[{ }^{3} \mathrm{H}\right]$ leucine injection and ipsilateral to that receiving the HRP injection. The extent of overlap at each site was determined by measuring directly the area 


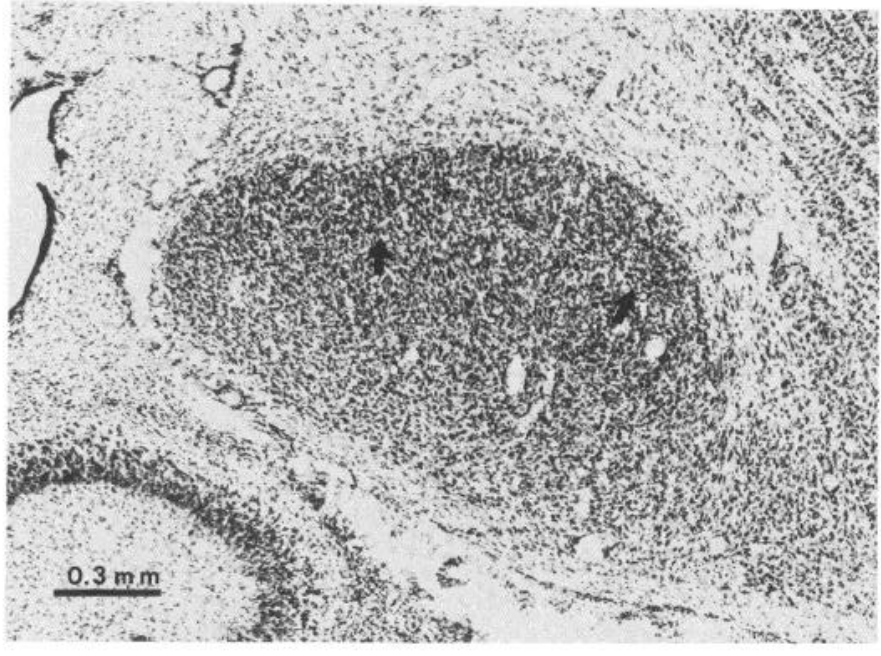

Figure 9. A higher power view of the left LGN in a section near that of Figure $8, A$ and $B$ to show the beginnings of an interlaminar region (between arrows) separating future layers $\mathrm{A}$ and $\mathrm{A} 1$ at E60. Cresyl violet-stained $20-\mu \mathrm{m}$ thick horizontal section. Medial is to the right; anterior is $u p$.

in which the two labels coincided (indicated in black in Fig. 13) and then expressing it as a percentage (shown in Fig. 13) of the total cross-sectional LGN area in each drawing.

Several important points emerge from these doublelabel experiments. First is the general observation that, initially, there is a gradual increase in the percentage of the nucleus occupied simultaneously by both sets of afferents until sometime between E47 and E54, when overlap begins to fall toward postnatal levels. Note, however, that at each age shown in Figure 13 there is a consistent difference in the percentage of overlap measured on the two sides. Overlap is always greater on the left side: contralateral to the eye injection of $\left[{ }^{3} \mathrm{H}\right]$ leucine and ipsilateral to the HRP injection. This result is not surprising in view of the possibility, discussed under "Materials and Methods," that spillover may contribute to the autoradiographic labeling pattern, in which case spillover would be expected to make a larger contribution contralaterally.

Progressive changes in the pattern of overlap can also be seen in Figure 13. For instance, overlap within areas of the nucleus destined to become the $\mathrm{C}$ layers persists beyond that in future A layer regions. Similarly, overlap first decreases at the posteromedial pole of the nucleus (Fig. 13, E47) (future representation of the central visual fields) followed next by that further laterally at later ages (Figure 13, E60 and P2).

Perhaps the most remarkable finding is that there was no time during development when afferents from the two eyes overlapped each other evenly everywhere within the LGN (i.e., overlap $=100 \%$ ). Indeed, the area of coextensive labeling was at most $39 \%$ at E47 (Figure 12, E47: left), with the remaining $60 \%$ of the nucleus occupied primarily by afferents from one eye or the other. Inspection of Figure 13 reveals that at E47, of the remaining singly innervated territories, contralateral eye afferents predominate within much of the region destined to be- come future layer $\mathrm{A}$. This is the case not only at E47, but at every age, indicating that this particular territory may never be shared with ipsilateral eye afferents, a suggestion with many implications for any mechanistic explanation of the segregation process (see "Discussion").

By means of the second, more indirect method for estimating overlap from singly labeled material, it was possible to confirm and extend the above findings. The fisrt step was to determine the percentage of the area of the nucleus occupied by label both contralateral and ipsilateral to each eye injection in a larger series of animals (including those studied above: see "Materials and Methods"). As shown in Figure 14, measurements for the adult give the reasonable result that roughly $60 \%$ of the LGN is occupied by afferents from the contralateral eye (Fig. 14, solid circles), while about $30 \%$ is occupied by those from the ipsilateral eye (Fig. 14, open circles); the remaining $10 \%$ corresponds to the afferentfree interlaminar zones. On the other hand, at E32 the contralateral eye afferents occupy $100 \%$ of the LGN anlage; those from the ipsilateral eye have yet to invade the nucleus, hence $0 \%$ in Figure 14.

The period between $\mathrm{E} 32$ and $\mathrm{P} 2$, when the adult percentages have nearly been reached (Fig. 14), is characterized by systematic changes in the proportion of LGN area occupied by the two sets of afferents, as can be appreciated graphically in Figure 14. During this time contralateral eye afferents undergo a simple gradual reduction in the proportion of the nucleus they occupy from $100 \%$ to $60 \%$. In contrast, those from the ipsilateral eye never achieve more than a $55 \%$ occupancy of the nucleus, which then also gradually declines to $30 \%$ with a time course rather similar to that seen contralaterally. It is probably significant that the period of peak ipsilateral occupancy at E47 coincides with the initial fall from $100 \%$ to $85 \%$ in contralateral occupancy, a fall that corresponds anatomically to the first appearance of gaps in the contralateral labeling pattern (see Fig. 8 and 13,E47).

The results of Figure 14 were next used to estimate overlap indirectly by adding together the ipsilateral and contralateral percentages for each case and taking overlap, the percentage of the area of the nucleus innervated by both sets of afferents, to equal the amount by which the sum exceeded $100 \%$ (see "Materials and Methods"). This approach provided an extremely useful graphical record of the progressive changes in extent of overlap with age and therefore also of the time course of segregation. As shown in Figure 15, overlap first increases from $0 \%$ at E30 to a maximum average value of $33 \%$ ( \pm $8 \% \mathrm{SD}$ ) at $\mathrm{E} 47$ as afferents from the ipsilateral eye invade the already contralaterally innervated nucleus. Then, between E47 and birth, the degree of dual innervation (overlap) again falls to $0 \%$ as the two sets of afferents segregate completely from each other into their adultlike laminar pattern. (The negative overlap values present at P2 and in the adult simply indicate that at these ages up to $10 \%$ of the nucleus is composed of afferentfree interlaminar zones.) These indirect estimates of overlap correspond well with those measured directly in Figure 13, as can be appreciated from Table II, in which overlap values obtained both ways from the same cases are compared. Thus the results from both methods indicate that during prenatal development, afferents from 

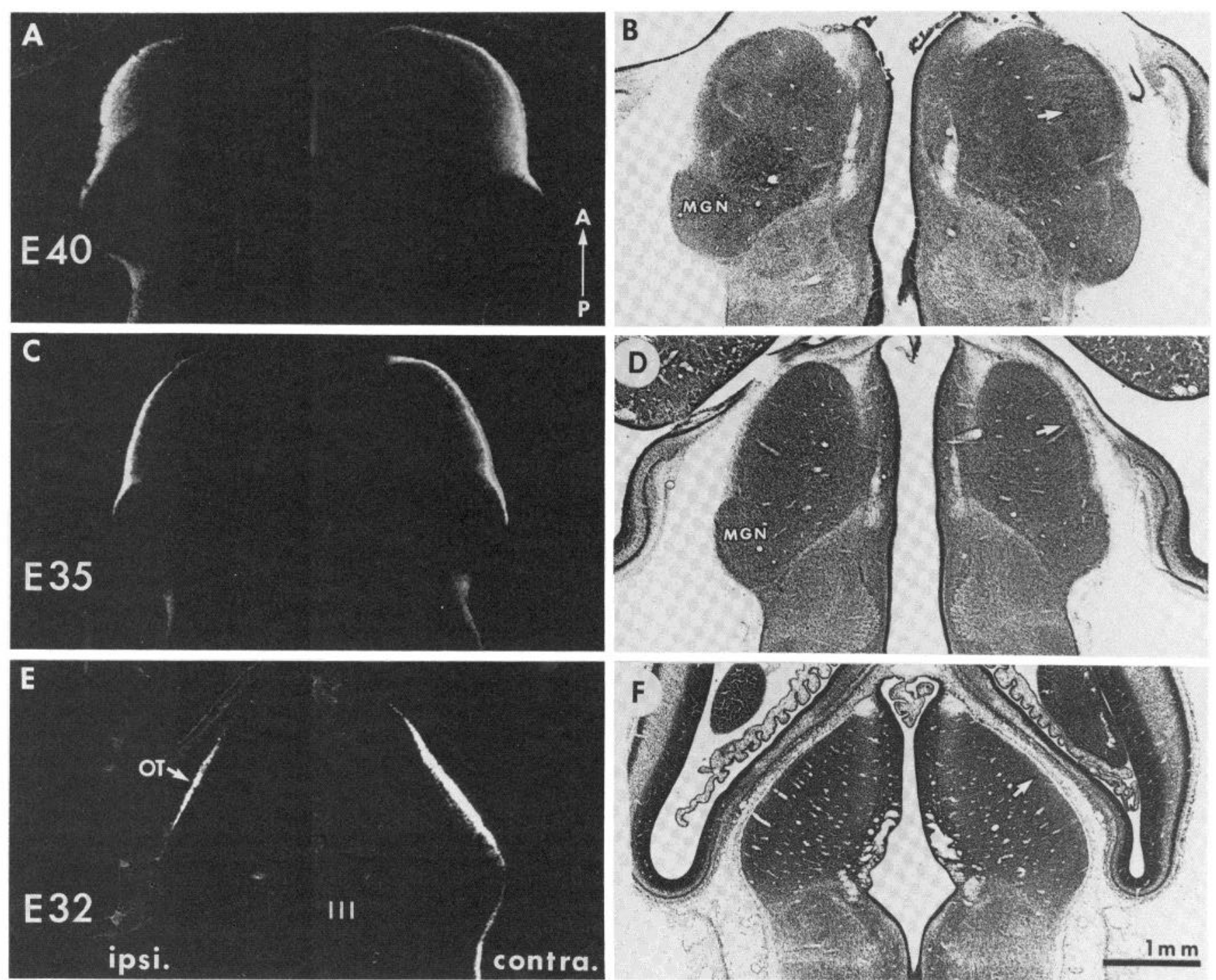

Figure 10. The retinogeniculate projection as seen in the horizontal plane in three fetuses aged E40 $(A$ and $B)$, E35 $(C$ and $D)$, and E32 $(E$ and $F)$. On the left are autoradiographs, in dark-field, of the distribution of labeled afferents following an intraocular injection of ${ }^{3} \mathrm{H}$-amino acids. At each age, projection to the LGN consists of thin bands of label crowning the anterior and lateral edge of the thalamus. Label, present posterior to the medial geniculate nucleus (see $B$, D:MGN) is contained within retinal afferents en route to the superior colliculus via the brachium of the superior colliculus. Note that at E32 $(E)$, the ipsilateral projection is confined to the optic tract $(O T)$, while at E35 $(C)$, a small finger of ipsilateral afferents has invaded the LGN at its posterior pole. On the right are the adjacent cresyl violet-stained sections to show the histological picture. In $B, D$, and $F$, the white arrow indicates the approximate location of the medial (inner) border of the LGN anlage (see the text for further details concerning this point). The calibration bar $(1 \mathrm{~mm})$ in $F$ refers to $A$ to $F$.

the two eyes share only about $40 \%$ of the total available territory within the LGN.

\section{Discussion}

This study has shown that the cat's retinogeniculate pathway develops during the second half of its 65-day gestation period. As in mammals, here, too, development is characterized by an initial period of overlap in which retinal afferents from the two eyes are intermixed in the LGN, followed secondarily by a period of segregation during which the two sets of afferents sort out from each other to produce the adult-like laminar pattern of input by birth. However, closer quantitative examination has revealed that throughout the period of overlap, intermixing is not complete: the contralateral eye retains primary control of territory within the LGN. In this regard, de- velopment in the cat is distinctly different from that in the rhesus monkey, in which it has been reported that LGN territory is completely shared by the afferents from both eyes prior to the onset of segregation (Rakic, 1977a). On the contrary, the extent of overlap seen within the cat's LGN during development is evidently somewhat less than that of another carnivore, the ferret (Linden et al., 1981), but a good deal more than that seen in rabbits (Ostrach et al., 1981) and rodents (So et al., 1978; Bunt et al., 1981).

Timing of development. By means of the anterograde transport methods used here it has been possible to revise downward the time that retinal afferents definitely invade the LGN from E37 (determined with degeneration techniques; Anker, 1977) to E32. At this age, the invasion consists almost entirely of afferents from the contralat- 

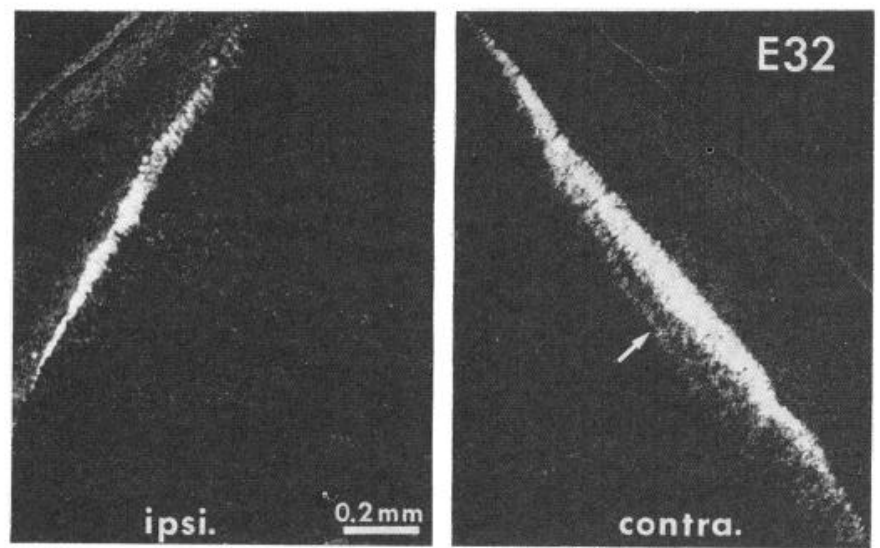

Figure 11. A high power view of the dark-field autoradiograph of Figure $9 E$ to show that at E32, afferents from the contralateral retina have already begun to invade the anlage of the LGN (arrow), while those from the ipsilateral eye are still confined largely to the optic tract. Anterior is up; posterior is down. eral eye, with those from the ipsilateral eye delayed several days longer. This delay, also seen in development of the rodent retinogeniculate pathway (So et al., 1978; Lund and Bunt, 1976; Land and Lund, 1979; Bunt et al., 1981) and thought to occur in the ferret as well (Linden et al., 1981; Cucchiaro and Guillery, 1982), may account for the ability later on of contralateral eye afferents to occupy exclusively a good deal of LGN territory in these species. It would be of great interest in this context to know whether a similar delay occurs in primates or whether, instead, afferents from both eyes invade the LGN in concert.

If early arriving afferents are to retain an edge over those coming later, some territory within the LGN must be available for immediate claim. The results of $\left[{ }^{3} \mathrm{H}\right]$ thymidine labeling experiments currently in progress show that this may very well be the case. Neurons comprising the LGN are first generated on E24 (Hickey and Cox, 1979; Shatz, 1981) and by E29 many of these have already migrated laterally to the margin of the
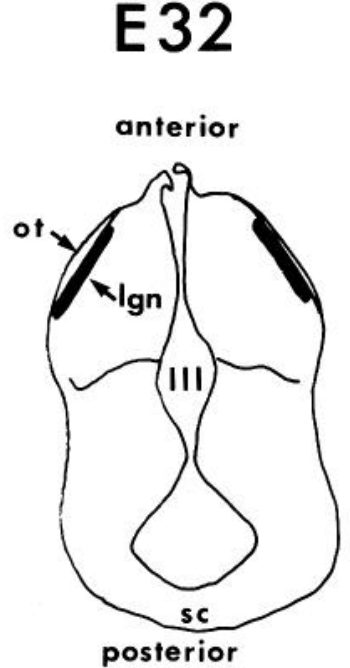

E 40

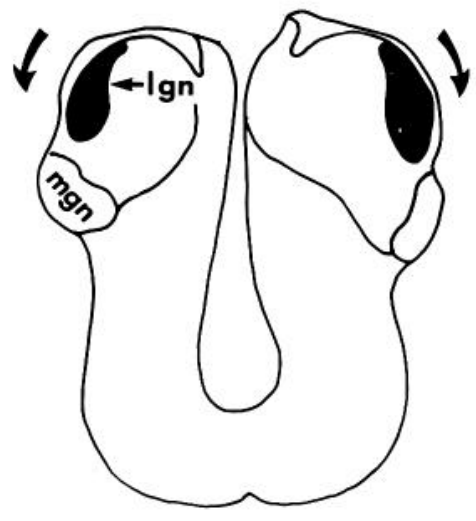

E 47

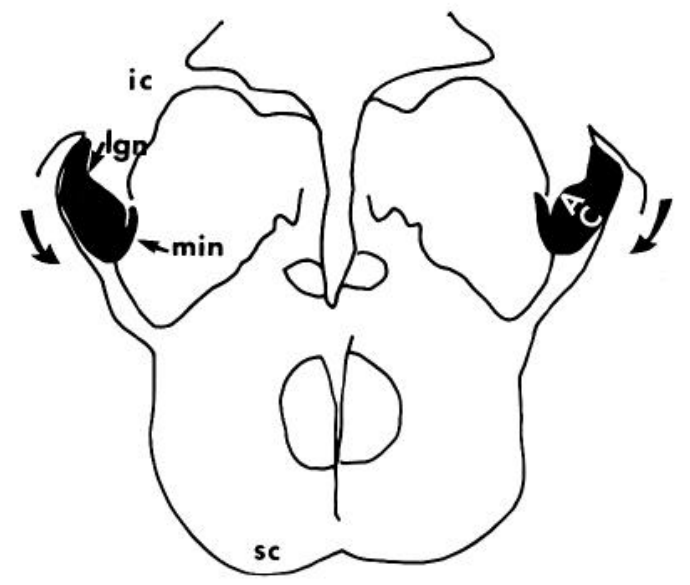

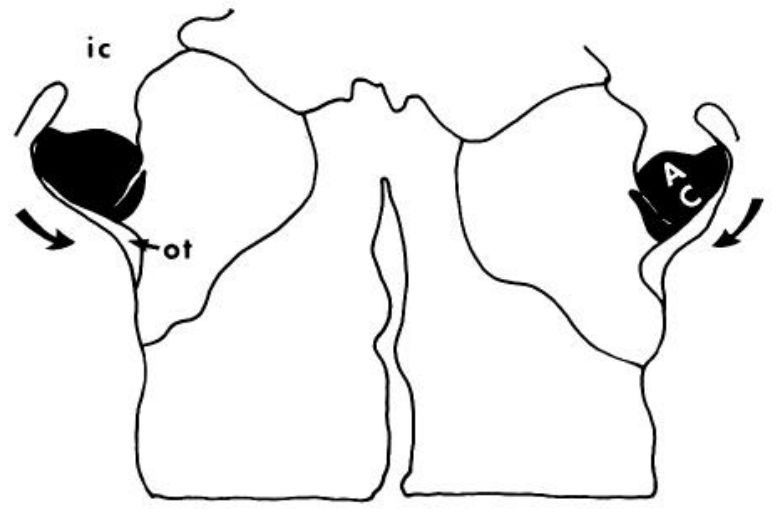

E54

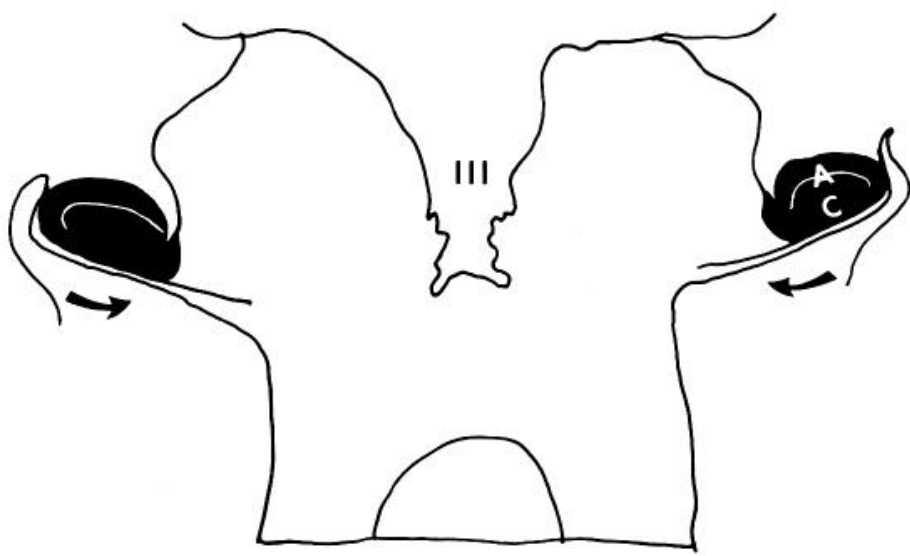

E60

$2 \mathrm{~mm}$

Figure 12. A series of camera lucida drawings of horizontal sections similar to those of Figures 8 and 9 to show the progressive displacement of the LGN (shown in black) during prenatal life. Between E40 and E60, the LGN shifts roughly $90^{\circ}$ in the horizontal plane, thereby displacing the E40 anterior pole laterally by E60. III, third ventricle; mgn, medial geniculate nucleus; $i c$, internal capsule; $s c$, superior colliculus; $\min$, medial interlaminar nucleus. $A$ represents the approximate location of laminae A and $\mathrm{A} 1 ; C$ represents the approximate position of the three $\mathrm{C}$ laminae. The calibration bar ( $2 \mathrm{~mm})$ is for all drawings. 

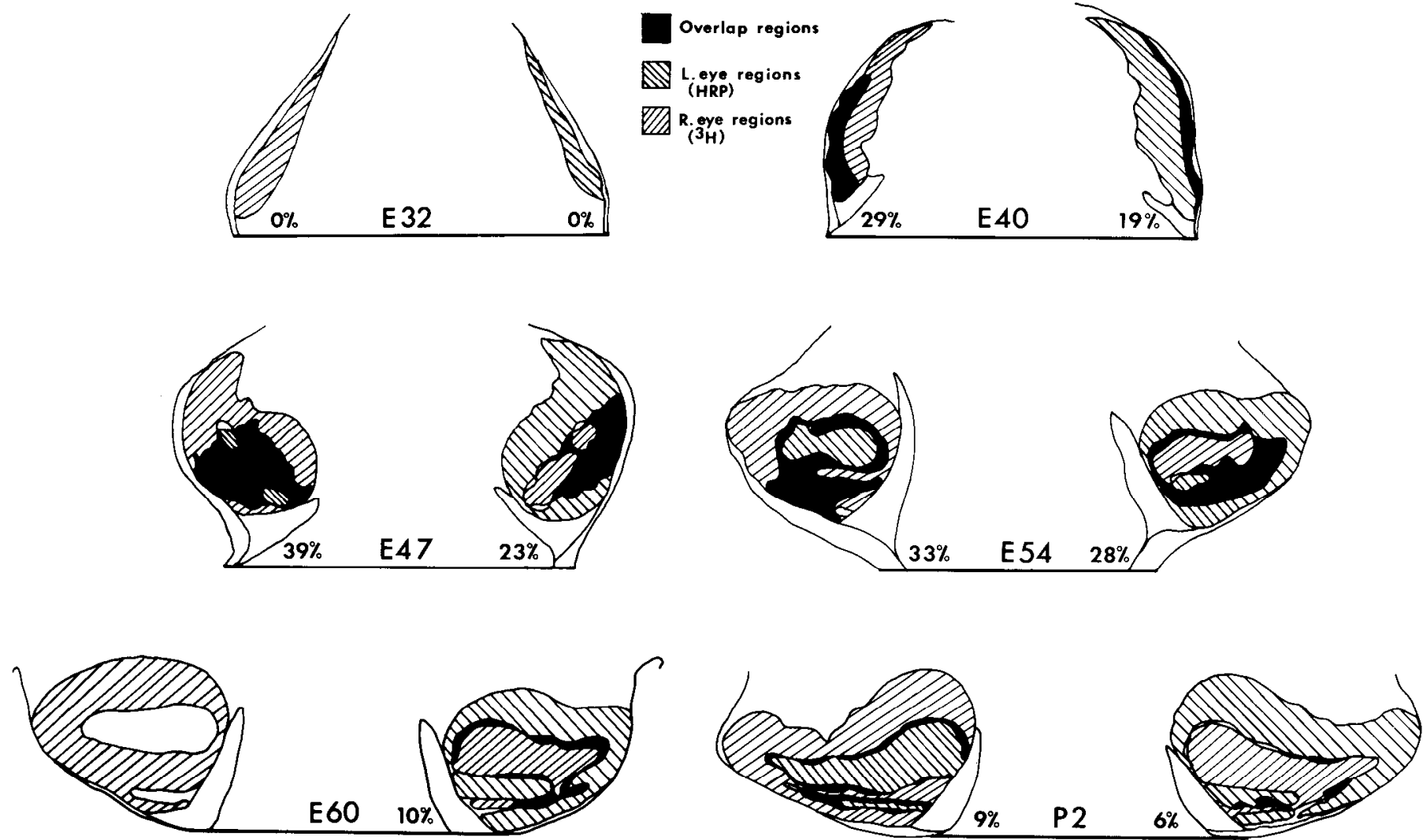

Figure 13. Camera lucida drawings of horizontal sections through the LGNs of a series of six animals to illustrate the progressive change in overlap of retinal afferents within the nucleus by means of double-label experiments. Each animal had received intraocular injections of HRP into the left eye and ${ }^{3} \mathrm{H}$-amino acids into the right eye. At each age, cross-hatching indicates the location of afferents containing either radioactive $\left({ }^{3} \mathrm{H}\right)$ or peroxidase (HRP) label; dually labeled areas are filled in with black. (Label within the optic tracts, perigeniculate nucleus, ventral LGN, and MIN was excluded.) Percentages adjacent to each LGN represent the area of overlap measured within each nucleus and are expressed as a percentage of total cross-sectional LGN area. At E32 and E40, total LGN area was set equal to the area innervated by the contralateral eye. Note that at E32, overlap is $0 \%$ because only afferents from the contralateral eye have invaded the LGN at this age. At E60, overlap measurements could only be obtained from the right LGN due to incomplete peroxidase labeling ipsilateral to the eye injection. See the text for further details.

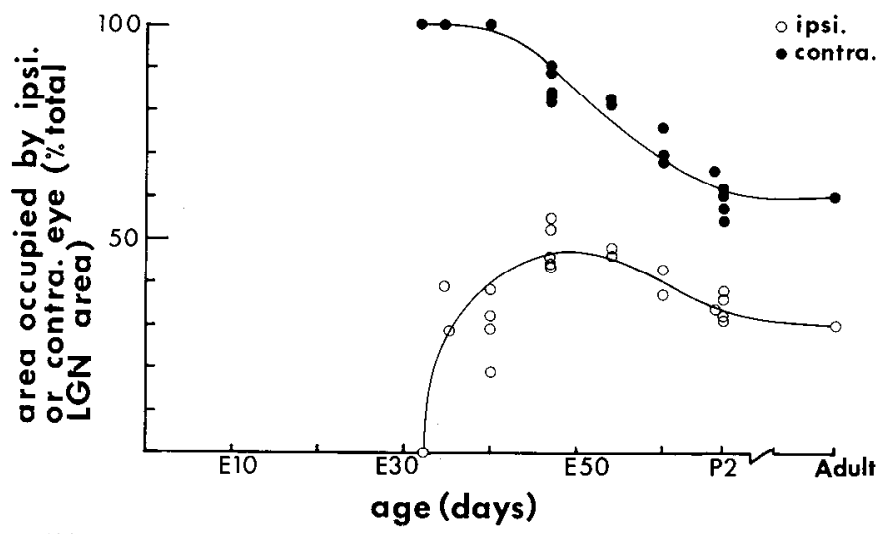

Figure 14. Progressive changes with age in areas occupied by afferents from the contralateral $(\bigcirc)$ and ipsilateral $(O)$ eyes as determined from the single-label experiments described in the text. Each point represents measurements from six to eight sections per animal in which the area occupied by the ipsilateral or contralateral eye afferents in each section was added and expressed as a percentage of total cross-sectional LGN area. (Curves were drawn by eye.) See the text for further details.

dorsal thalamus in close proximity to the optic tract. Similarly, the earliest retinal ganglion cells are generated by E21 (Polley et al., 1981; Kliot and Shatz, 1982) and the label seen within the optic lract at E28 following the

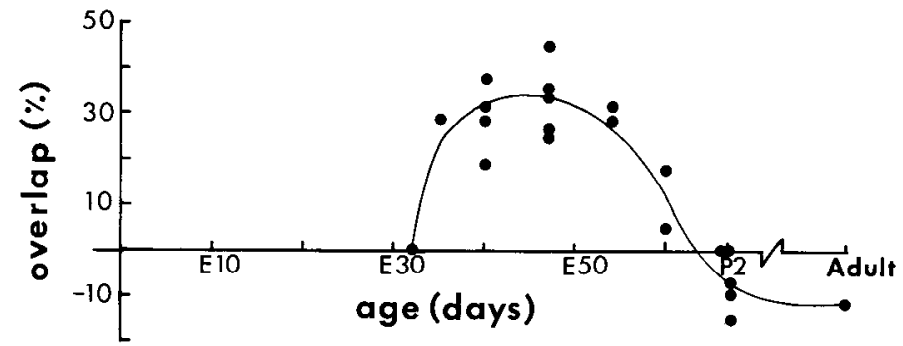

Figure 15. Age-related changes in extent of overlap between afferents from the two eyes within the LGN as determined indirectly from results of the single-label experiments (sec the text for complete details). Overlap here is a measure of the percentage of the nucleus occupied dually by afferents from both eyes. Each point represents a determination for a single animal in which overlap was set equal to the amount by which the sum of the ipsilateral and contralateral areal percentages obtained in Figure 14 exceeded 100\%. Negative overlap values indicate that some regions of the nucleus are completely free from input from either eye. (Curve was drawn by eye.)

first successful eye injections is presumably containcd within their axons. These timing relationships make it quite possible for the first set of retinal afferents reaching the vicinity of the LGN to confront their target neurons immediately. The alternative, that the afferents undergo a "waiting period" similar to that found in the develop- 
TABLE II

Comparison of overlap estimates obtained by means of the two methods described in the text

Note that at each age, measurements refer to one animal that was doubly labeled.

\begin{tabular}{ccc}
\hline \multirow{2}{*}{ Age } & \multicolumn{2}{c}{ Method Used } \\
\cline { 2 - 3 } & Double label (Fig. 12) & Single label (Fig. 14) \\
\hline \multirow{2}{*}{ P2 } & 6 & $\%$ \\
& 9 & $-7(\mathrm{HRP})^{a}$ \\
E60 & 10 & $0\left({ }^{3} \mathrm{H}\right)^{a}$ \\
E54 & 28 & $18\left({ }^{3} \mathrm{H}\right)$ \\
& 33 & $32(\mathrm{HRP})$ \\
E47 & 23 & $29\left({ }^{3} \mathrm{H}\right)$ \\
& 39 & $45(\mathrm{HRP})$ \\
E40 & 19 & $34\left({ }^{3} \mathrm{H}\right)$ \\
& 29 & $19(\mathrm{HRP})$ \\
E35 & $-{ }^{b}$ & $29\left({ }^{3} \mathrm{H}\right)$ \\
E32 & 0 & $29\left({ }^{3} \mathrm{H}\right)$ \\
& & 0
\end{tabular}

"The type of label, HRP or "H-animo acids, used to obtain the estimate is indicated in parentheses.

${ }^{b}-$, not done.

ment of the geniculocortical pathway in other species (Rakic, 1977a; Lund and Mustari, 1977), is much less likely in view of results from these $\left[{ }^{3} \mathrm{H}\right]$ thymidine studies.

In thinking about the development of the retinogeniculate pathway, it is worth bearing in mind that during the period between E32, when the first contralateral afferents enter the LGN anlage, and E47, when maximal overlap between the ipsilateral and contralateral afferents is achieved, a dynamic relationship must exist between the retinal afferents and their postsynaptic targets: the LGN neurons. This period is characterized by the constant addition, through migration, of LGN neurons (Shatz, 1981) and, through axonal growth, of retinal afferents supplied by progressively later-generated ganglion cells, the last of which are born by E46 in the far periphery of the retina (Kliot and Shatz, 1982). The situation after E46, in contrast, is probably quite different: segregation of the two sets of afferents operates against the background of a largely nonmigratory postsynaptic population. Even at this age, however, the relationship between the retinal afferents and their postsynaptic targets may be far from stable. Recent studies have suggested that roughly $50 \%$ of retinal ganglion cells in the cat are eliminated by cell death possibly between F.46 and F.57 ( $\mathrm{Ng}$ and Stone, 1982; Stone et al., 1982; Williams et al., 1983). It remains to be determined whether a similar phenomenon occurs within the LGN and, if so, exactly how and when this process contributes to the final pattern of retinogeniculate connectivity seen by birth.

Displacement of the LGN and topography. Embryologists have long known that during development, differential growth of the mammalian brain can result in profound alterations in the position and alignment of particular structures. Among the best modern examples is the LGN of higher mammals. For instance, the monkey's LGN is displaced ventrally and laterally in the coronal plane during prenatal development (Rakic, 1977b). Similarly, Kalil (1978) has shown that between birth and about 4 months postnatally the cat's LGN undergoes a major axial shift of $90^{\circ}$ in the sagittal plane that brings ventral regions anterior and dorsal regions posterior. Results of the present study show that this postnatal shift is preceded prenatally by another largely separate $90^{\circ}$ displacement, this time in the horizontal plane, occurring between E40 and E60. The onset of this displacement coincides nicely with the time that the majority of migrating neurons have reached the vicinity of the LGN anlage and also with the time that a major projection from the retina has already been established. Moreover, by E40, the LGN neurons themselves have begun to elaborate axonal connections with the telencephalon (Luskin and Shatz, 1982), and by 1 week later, if not earlier, the first corticogeniculate connections have arrived within the vicinity of the LGN (Anker, 1977). Thus, this displacement creates no special logistical complications for the development of the retinogeniculocortical pathways.

Although virtually nothing is presently known concerning the development of topographic order in the cat's retinogeniculate projection, several broad inferences can be made from the above considerations. One has to do with the general orientation of the retinotopic map within the nucleus prior to the onset of LGN displacement. It is highly likely that, initially, the central retina is mapped at the posterior pole, while the peripheral retina is mapped at the anterior pole of the LGN. This orientation is similar to that of the adult monkey's LGN (Malpeli and Baker, 1975). As for the other retinal axis, the superior retina would be expected to project ventrally and the inferior retina, dorsally in the cat embryo. Such an alignment of the retinotopic map initially is the only one possible given both the observed displacements of the nucleus in the horizontal and sagittal planes and the alignment of the retinotopic map present in the adult LGN (Sanderson, 1971). It is also consistent with the emerging laminar pattern of afferent input to the LGN seen between E47 and E60 as segregation proceeds. Thus, it may be that the original orientation of the retinotopic map within the cat's and monkey's LGN is identical, but that subsequent displacements in different planes (cf. Rakic, 1977b) give rise to the differing alignments of the retinotopic maps seen in the adult.

The process of segregation. A major goal of this study was to obtain information concerning the time course and pattern of segregation of eye input to the cat's LGN as a necessary preface to further studies of the segregation process. In many respects, the timing of segregation seen here in the cat is very similar in its general features to that found in other species in which afferent input from the two eyes is also highly segregated in the adult. For instance, the onset of afferent segregation in the nucleus has been found to precede the initiation of cellular lamination in the ferret (Linden et al., 1981), tree shrew (Brunso-Bechtold and Casagrande, 1981), and monkey (Rakic; 1977b; P. Rakic, personal communication), and the cat is no exception: segregation begins at $\mathrm{E} 47$, roughly 2 weeks before cellular lamination can first be detected. The material available here, however, is not ideally suited for a precise determination of the time course of lamination since the requirements of peroxidase histochemistry necessitated the use of thick (20 to 50 
$\mu \mathrm{m})$ sections. Thus, it is conceivable that cellular lamination occurs somewhat earlier than seen in this study. Finally, the completion of segregation of retinogeniculate afferents in the cat, as in the monkey, occurs roughly 3 weeks before segregation of the geniculocortical afferents begins at the cortical level (Rakic, 1977b; LeVay et al., 1978).

Not surprisingly, many details of segregation within the cat's LGN are consistent with the characteristic laminar organization found in the adult and therefore differ from those seen in other species with the exception of its close relative the ferret (Linden et al., 1981). In the adult cat, the LGN can be subdivided both functionally and anatomically into the A laminae (A and A1) and the C laminae (C, C1, C2) and it is now known that each subdivision ( $\mathrm{A}$ or $\mathrm{C}$ ) receives projections from different classes of retinal ganglion cell axons (for review see Guillery, 1979). It is tempting to view the finding here that segregation of afferents into laminae $\mathrm{A}$ and $\mathrm{A} 1$ occurs before segregation in the $\mathrm{C}$ laminae as an indication that each class of retinal ganglion cell axon may develop with a different time course. This suggestion is consistent with preliminary $\left[{ }^{3} \mathrm{H}\right]$ thymidine studies of the retina in which differences in the initiation and duration of genesis of different populations of ganglion cells have been found (Polley et al., 1981; Kliot and Shatz, 1982). Although the birthdates of retinal ganglion cells and the final identity and position of their axons are currently unknown, the issue is central to a thorough understanding of visual system development and serves to underscore the clegant complexity of the process under consideration here.

It was significant to find that, prior to the onset of segregation, afferents from the contralateral eye retain principal control of some territory within the LGN, in particular a portion of lamina $\mathrm{A}$, throughout prenatal development in the cat. In contrast, prior to segregation, overlap of the afferents is "almost complete" in the ferret LGN (Linden et al., 1981, p. 206) and evidently is complete in the monkey's LGN (Rakic, 1977a); thus the question arises as to whether there are genuine species differences or, instead, whether methodological differences may contribute to the findings. First, in regard to methodology, the accuracy of the overlap measurements made here depends directly on the determination of the borders between the dorsal lateral geniculate nucleus and its neighbors: the optic tract, MIN, ventral LGN, and perigeniculate nucleus. Each of these structures in the adult receives axonal projections from distinct subpopulations of retinal ganglion cells (for review, see Rodieck, 1979) and it is reasonable to think that during development the time course and pattern of afferent segregation within each is likely to differ from that of the dorsal IGN (This supposition is supported by Williams and Chalupa's study (1982) of the prenatal development of the cat's retinocollicular pathway, in which it was reported that extensive overlap occurs between contralateral and ipsilateral retinocollicular afferents prior to segregation.) Thus measurements were confined exclusively to dorsal LGN and it is worth emphasizing here that to do so reliably required the use of horizontal rather than coronal sections.
The significance of the overlap measurements also depends on the criteria used to distinguish labeled from unlabeled territory within the LGN. In this study, measurements were designed to obtain a graphic representation of the clear disparity in both the distribution and density of labeling seen between ipsilateral and contralateral eye input. Consequently, very sparsely labeled regions were considered unlabeled here, thereby possibly minimizing the percentage of overlap measured at each age. This factor could account for the difference noted between cat and ferret in degree of overlap, but it is difficult to reconcile that found between the cat and monkey without invoking some genuine species difference as well.

Perhaps the most compelling evidence favoring partial overlap comes from the direct measurements made in the double-labeled material in which one eye had been injected with HRP and the other with $\left[{ }^{3} \mathrm{H}\right]$ leucine. It is conceivable that overlap might have been underestimated from the distribution of HRP reaction product if it were to fail to fill the finest terminals, or overestimated from the autoradiographic labeling pattern due to spillover of label from fibers and terminals (see "Materials and Methods" and LcVay ct al., 1978). Taken together, however, the results provide reasonable lower and upper limits for the extent of overlap at each age. These limits, admittedly approximate at best, are always significantly less than $100 \%$ overlap. Nevertheless, more accurate quantitative information is clearly desirable and we (C. J. Shatz and M. Siegel, manuscript in preparation) are currently in the process of collecting it at the electron microscopic level where quantitation and the unambiguous identification of terminals and fibers of passage are considerably more feasible.

If overlap of both sets of afferents within the cat's LGN is never complete, then it is necessary to revise somewhat the notion that segregation is achieved through equal and simultaneous competition between the afferents from both eyes for territory throughout the entire LGN (see Purves and Lichtman, 1980, for review). Some form of competition is in operation at the level of the LGN, since removal of one eye during prenatal life does affect the degree of segregation attained by the remaining sets of afferents (Rakic, 1981; Williams et al., 1982). However, results here suggest that competition may be perturbed initially in favor of afferents from the contralateral eye, possibly by virtue of their early arrival, thereby allowing these afferents to retain control of lamina A throughout development. The requirements of controlling territory within lamina A might subsequently place the contralateral eye afferents at a competitive disadvantage within future lamina $\mathrm{A} 1$, permitting the final takeover there by ipsilateral eye afferents. Similar considerations could apply to segregation within the $C$ laminae. One corollary of this model of biased competition is that the same laminar sequence of an eye input would always be produced in the adult, a necessary prerequisite of any adequate explanation of the segregation process at the geniculate, but not necessarily at the cortical, level. (Even at the cortical level, it is worth pointing out that exclusively monocular regions do exist at the representations of the optic disc and monocular 
segment and could act in a similar way during the formation of ocular dominance columns to perturb initially the competitive process.) Another related issue concerns the nature of the competitive interactions occurring between the afferents during embryonic life. In other systems in which segregation occurs during development postnatally and in the peripheral nervous system, competition is thought to involve the formation and breakage of functioning synaptic connections (Purves and Lichtman, 1980) and the question naturally arises whether such interactions actually occur within the fetal central nervous system. In an attempt to further understand the phenomenon of segregation as exemplified in the development of the cat's retinogeniculate pathway, we (Shatz et al., 1982) are currently investigaling the possibilities raised here at the level of the electron microscope and by means of a fetal electrophysiology.

\section{References}

Anker, R. L. (1977) The prenatal development of some visual pathways in cat. J. Comp. Neurol. 173: 185-204.

Brunso-Bechtold, J. K., and V. A. Casagrande (1981) Effect of bilateral enucleation on the development of layers in the dorsal lateral geniculate nucleus. Neuroscience $6: 2579-2586$.

Bunt, S., R. D. lund, and P. W. Land (1981) The prenatal development of the primary optic projection in hooded and albino rats. Invest. Ophthalmol. Vis. Sci. (Suppl.) 20: 174.

Cucchiaro, J., and R. W. Guillery (1982) The structure and development of the dorsal lateral geniculate nucleus and its retinal afferents in the albino ferret (Mustela putorius furo). Soc. Neurosci. Abstr. 8: 811.

Frank, E., W. A. Harris, and M. B. Kennedy (1980) Lysophosphatidyl choline facilitates labeling of CNS projections with horseradish peroxidase. J. Neurosci. Methods 2: 183-189.

Guillery, R. W. (1970) The laminar distribution of retinal fibers in the dorsal lateral geniculate nucleus of the cat: $A$ new interpretation. J. Comp. Neurol. 138: 339-368.

Guillery, R. W. (1979) A speculative essay on geniculate lamination and its development. Prog. Brain Res. 51: 403-418.

Hayhow, W. R. (1958) The cytoarchitecture of the lateral geniculate body in relation to the distribution of crossed and uncrossed fibers. J. Comp. Neurol. 110: 1-51.

Hickey, T. L., and N. R. Cox (1979) Cell birth in the lateral geniculate nucleus of the cat: A ${ }^{3} \mathrm{H}$-thymidine study. Soc. Neurosci. Abstr. 5: 788.

Hickey, T. L., and R. W. Guillery (1973) An autoradiographic study of retinogeniculate pathways in the cat and fox. J. Comp. Neurol. 156: 239-253.

Hubel, D. H., T. N. Wiesel, and S. LeVay (1977) Plasticity of ocular dominance columns in monkey striate cortex. Philos. Trans. R. Soc. Lond. (Biol.) 278: 377-409.

Kalil, R. (1978) Development of the dorsal lateral geniculate nucleus in the cat. J. Comp. Neurol. 182: 265-292.

Kliot, M., and C. J. Shatz (1982) Genesis of ganglion cells in the cat's retina. Soc. Neurosci. Abstr. 8: 815.

Land, P. W., and R. D. Lund (1979) Development of the rat's uncrossed retinotectal pathway and its relation to plasticity studies. Science 205: 698-700.

T.eVay, S., M. P. Stryker, and C. J. Shatz (1978) Ocular dominance columns and their development in layer IV of the cat's visual cortex: A quantitative study. J. Comp. Neurol. 179: 223-244.

LeVay, S., T. N. Wiesel, and D. H. Hubel (1980) The development of ocular dominance columns in normal and visually deprived monkeys. J. Comp. Neurol. 191: 1-51.
Linden, D. C., R. W. Guillery, and J. Cucchiaro (1981) The dorsal lateral geniculate of the normal ferret and its postnatal development. J. Comp. Neurol. 203: 189-211.

Lund, R. D., and A. H. Bunt (1976) Prenatal development of central optic pathways in albino rats. J. Comp. Neurol. 165: 247-264.

Lund, R. D., and M. J. Mustari (1977) Development of the geniculocortical pathway in rats. J. Comp. Neurol. 173: 289306.

Luskin, M. B., and C. J. Shatz (1982) Neurogenesis of the cat's primary visual cortex. Soc. Neurosci. Abstr. 8: 3 .

Malpeli, J. G., and F. H. Baker (1975) The representation of the visual field in the lateral geniculate nucleus of Macaca mulatta. J. Comp. Neurol. 161: 569-594.

McCance, I., J. W. Phyllis, and B. Wright (1966) The seriatim removal of foetal kittens. J. Reprod. Fertil. 12: 229-232.

Mesulam, M. M. (1976) The blue reaction product in horseradish peroxidase neurohistochemistry: Incubation parameters and visibility. J. Histochem. Cytochem. 24: 1273-1280.

Minkowski, M. (1920) Über den verlauf, die endigung, und die zentrale repräsentation von gekreuzten und ungekrenzten sehnerventasern bei einigen säugethieren und bein menschen. Schweiz. Arch. Neurol. Psychiatr. 6: 201-252.

$\mathrm{Ng}, \mathrm{A} . \mathrm{Y} . \mathrm{K}$., and J. Stone (1982) The optic nerve of the cat: Appearance and loss of axons during normal development. Dev. Brain Res. 5: 263-271.

Ostrach, L. H., K. L. Chow, and J. W. Crabtree (1981) Postnatal development of ipsilateral retinotectal and retinogeniculate projections in the rabbit. Invest. Ophthalmol. Vis. Sci. (Suppl.) 20: 73.

Polley, E. H., C. Walsh, and T. L. Hickey (1981) Neurogenesis in cat retina. A study using ${ }^{3} \mathrm{H}$-thymidine autoradiography. Soc. Neurosci. Abstr. 7: 672 .

Purves, D., and J. W. Lichtman (1980) Elimination of synapses in the developing nervous system. Science 210: 153-157.

Rakic, P. (1976) Prenatal genesis of connections subserving ocular dominance in the rhesus monkey. Nature 261: 467471.

Rakic, P. (1977a) Prenatal development of the visual system in rhesus monkey. Philos. Trans. R. Soc. Lond. (Biol.) 278: 245260.

Rakic, P. (1977b) Genesis of the dorsal lateral geniculate nucleus in the rhesus monkey: Site and time of origin, kinetics of proliferation, routes of migration and pattern of distribution of neurons. J. Comp. Neurol. 176: 23-52.

Rakic, P. (1979) Genesis of visual connections in the rhesus monkey. In Developmental Neurobiology of Vision, R. D. Freeman, ed., pp. 249-260, Plenum Publishing Corp., New York.

Rakic, P. (1981) Development of visual centers in the primate brain depends on binocular competition before birth. Science 214: $928-931$.

Rodieck, R. W. (1979) Visual pathways. Annu. Rev. Neurosci. 2: $193-225$.

Sanderson, K. (1971) The projection of the visual field to the lateral geniculate and medial interlaminar nuclei in the cat. J. Comp. Neurol. 143: 101-118.

Scott, P. P. (1970) Cats. In Reproduction and Breeding Techniques for Laboratory Animals, E. S. E. Hafez, ed., pp. $192-$ 208, Lea and Febiger, Philadelphia.

Shatz, C. J. (1981) Inside-out pattern of neurogenesis of the cat's lateral geniculate nucleus. Soc. Neurosci. Abstr. 7: 140.

Shatz, C. J., and A. C. DiBerardino (1980) Prenatal development of the retinogeniculate pathway in the cat. Soc. Neurosci. Abstr. 6: 485.

Shatz, C. J., and M. Kliot (1982) Prenatal misrouting of the retinogeniculate pathway in the Siamese cat. Nature 300 : 525-529. 
Shatz, C. J., S. Lindstrom, and T. N. Wiesel (1977) The distribution of afferents representing the right and left eyes in the cat's visual cortex. Brain Res. 131: 103-116.

Shatz, C. J., P. A. Kirkwood, and M. W. Siegel (1982) Functional retinogeniculate synapses in fetal cats. Soc. Neurosci. Abstr. 8: 815 .

So, K. -F., G. E. Schneider, and D. O. Frost (1978) Postnatal development of retinal projections to the lateral geniculate body in Syrian hamsters. Brain Res. 142: 343-352.

Stone, J., D. H. Rapaport, R. W. Williams, and L. Chalupa (1982) Uniformity of cell distribution in the ganglion cell layer of prenatal cat retina: Implications for mechanisms of retinal development. Dev. Brain Res. 2: 231-242.

Williams, R. W., and L. M. Chalupa (1982) Prenatal development of retinocollicular projections in the cat: An anterograde tracer transport study. J. Neurosci. 2: 604-622.

Williams, R. W., M. J. Bastiani, and L. M. Chalupa (1983) Loss of axons in the cat optic nerve following fetal unilateral enucleation: An electron microscopic analysis. J. Neurosci. 3: 133-144.

Windle, W. R. (1935) Neurofibrillar development of cat embryos: Extent of development in the telencephalon and diencephalon up to $15 \mathrm{~mm}$. J. Comp. Neurol. 63: 139-171. 